\title{
Can you see the bubbles in a foam?
}

\author{
ÁRPÁD KuRUSA*
}

\begin{abstract}
An affirmative answer to the question in the title is proved in the plane by showing that any real analytic multicurve can be uniquely determined from its generalized visual angles given at every point of an open ring around the multicurve.
\end{abstract}

\section{Introduction}

Let a thin beam of X-rays travel along a straight line $\ell$ through a body. Let the density of the material of the body be described by the function $f$. Then, by physics, the line integral of $f$ along $\ell$ equals $\log \left(I_{0} / I\right)$, where $I_{0}$ and $I$, respectively, are the intensities of the beam before entering the body and after leaving it. In numerous tomographic devices this correlation and the inversion of the Radon transform constitutes the theoretic base of the unique determination of $f$ from the measurements of $\log \left(I_{0} / I\right)$ on a lot of straight lines. This widespread approach is based on the assumption that $f$ is regular enough. This is not the case if the body has a cellular structure with very thin cell wall, like a foam. For a foam the measured value $\log \left(I_{0} / I\right)$ on a straight line $\ell$ depends mostly only on the number of intersections where $\ell$ meets the membranes.

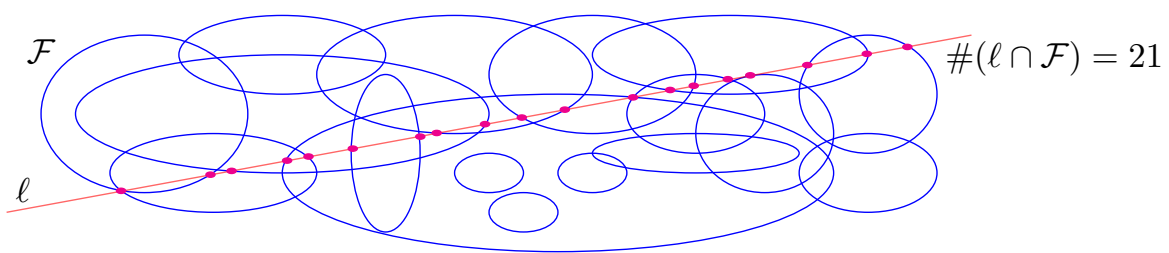

Figure 1.1. The line $\ell$ intersects the membrane of the foam $\mathcal{F}$ exactly 21 times

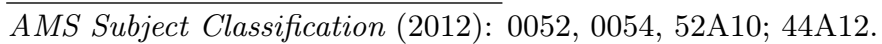

Key words and phrases: visual angle, masking function, Steinhaus, Crofton.

${ }^{*}$ This research was supported by National Research, Development and Innovation Office of Hungary (NKFIH), grant number NKFIH 116451. 
Such a measurement is clearly very unstable, and very unreliable, because any finite set of measurements can avoid small bubbles or just touch some membranes (like the one shown on Figure 1.1). To obviate this problem one can average those values for the straight lines passing through a point $P$ which, in turn, makes also physical sense: one just measures the X-radiation ${ }^{1}$ coming through the foam into the point $P$.

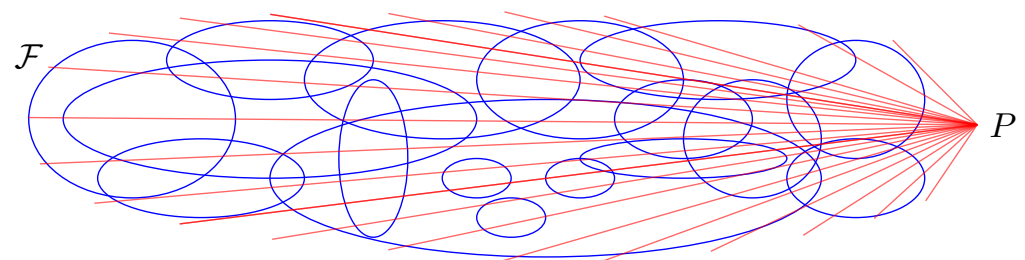

Figure 1.2. Measuring the $\mathrm{X}$-radiation coming through foam $\mathcal{F}$ to point $P$

Hence our question is: "Can the bubbles of a foam be uniquely determined by measuring X-radiation only at points outside of the foam?' In this article we give an affirmative answer to this question after making a suitable mathematical model.

A multicurve ${ }^{2} \boldsymbol{r}_{\mathcal{J}}$ is a finite set of parametrized curves, the members of the multicurve, $\boldsymbol{r}_{j}:\left[a_{j}, b_{j}\right] \rightarrow \mathbb{R}^{2}(j \in \mathcal{J})$ are of finite length and do not intersect each other in an open arc. A multicurve is said to have a property if each of its member curves satisfies that property. ${ }^{3}$

The trace $\operatorname{Tr} \boldsymbol{r}$ of a curve $\boldsymbol{r}:[a, b] \rightarrow \mathbb{R}^{2}$ is the set of points in $\mathbb{R}^{2}$ that is in the range of the function $\boldsymbol{r}$. The trace $\operatorname{Tr} \boldsymbol{r}_{\mathcal{J}}$ of a multicurve $\boldsymbol{r}_{\mathcal{J}}$ is the union $\bigcup_{j \in \mathcal{J}} \operatorname{Tr} \boldsymbol{r}_{j}$ of the traces $\operatorname{Tr} \boldsymbol{r}_{j}$ of the curves $\boldsymbol{r}_{j}$ of the multicurve $\boldsymbol{r}_{\mathcal{J}}$.

We denote the Grassman manifold of the straight lines in the plane by $\mathcal{L}$, and call a strictly positive continuous function $\bar{\omega}: \mathcal{L} \rightarrow \mathbb{R}_{+}$as line weight ${ }^{4}$. We define the weighted masking number $M_{\mathcal{T} ; \bar{\omega}}(P)$ of the trace $\mathcal{T}=\operatorname{Tr} \boldsymbol{r}_{\mathcal{J}}$ of the multicurve $\boldsymbol{r}_{\mathcal{J}}$ by

$$
M_{\mathcal{T} ; \bar{\omega}}(P)=\frac{1}{2} \int_{\mathbb{S}^{1}} \#(\mathcal{T} \cap \ell(P, \boldsymbol{w})) \bar{\omega}(\ell(P, \boldsymbol{w})) d \boldsymbol{w},
$$

where $\mathbb{S}^{1}$ is the unit circle, $\ell(P, \boldsymbol{w})$ is the straight line through the point $P \in \mathbb{R}^{2}$ with direction $\boldsymbol{w} \in \mathbb{S}^{1}$ and \# is the counting measure ${ }^{5}$.

\footnotetext{
${ }^{1}$ Instead of X-radiation normal light could also be used if the foam is translucent.

${ }^{2} \mathrm{~A}$ similar construction can be found in [17].

${ }^{3}$ With these terms a multisegment and a multicircle is a multicurve having only segments and circles, respectively, as members.

${ }^{4}$ Continuing the physical analogy given at Figure 1.2, a line weight could be thought of as a filter at the measuring points.

${ }^{5}$ In our considerations this number is finite at almost every point.
} 
The point $P$ and the set $\mathcal{T}$ are usually called the source and the object, respectively, of the masking number $M_{\mathcal{T} ; \bar{\omega}}(P)$, which is called the point projection by [3] and the shadow picture by [6] if $\mathcal{T}$ is convex. The function $M_{\mathcal{T} ; \bar{\omega}}: \mathbb{R}^{2} \rightarrow \mathbb{R}_{0 \leq}$ is called the weighted masking function of $\mathcal{T}$.
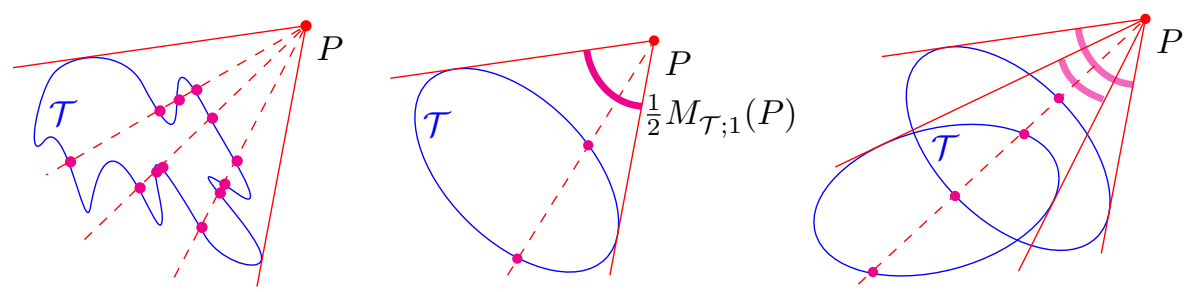

Figure 1.3. Observe that if $\mathcal{T}$ is a closed convex curve, then the measure of its visual angle at a point $P \notin \mathcal{T}$ is half of $M_{\mathcal{T} ; 1}(P)$; if $\mathcal{T}$ is a multicurve composed of more closed convex curves, then $M_{\mathcal{T} ; 1}(P)$ is the sum of the measures of the visual angles the convex curves subtend at $P \notin \mathcal{T}$.

We reformulate our question above as a problem to find such sets $\mathcal{S}$ of sources and sets $\mathcal{O}$ of objects that $\mathcal{O} \ni \mathcal{T} \mapsto M_{\mathcal{T}, \bar{\omega}} \mid \mathcal{S}$ is injective. There are numerous results of this kind for special multicurves in the literature: [6-8], [10-13], and also $[2,10,16]$ can be listed ${ }^{6}$ here. With these terms the author's original Conjecture in [10] takes the following more general form.

Conjecture. Given any line weight and two twice differentiable multicurves in the interior of a compact convex domain $\mathcal{D}$, the weighted masking functions of these multicurves are equal on an open ring around $\mathcal{D}$ if and only if the traces of the multicurves coincide.

In this article we only consider such line weights that depend exclusively on the directions of lines and call them $d$-weights for short.

In Section 2 we give all necessaries needed to investigate the masking function for twice differentiable curves of a natural class and establish an explicit formula for the masking function.

In Section 3 we first calculate the directional derivatives of the masking function, which generalizes a number of earlier results on visual angles given in [6-8] and [10-13]. At the end, the Laplacian of the masking function is given for d-weighted masking functions (Theorem 3.5).

${ }^{6}$ The cross integral in [2], the weighted back projection in [16] and the generalized visual angle in [10, Eq. (2) in Sect. 3] are in fact weighted masking functions with special weights. 
In Section 4 the results $^{7}$ [2, Theorem 10.2] and [16, Theorem 2.1] on the Steinhaus problem [19] are generalized to d-weights, although restricted to twice differentiable multicurves (see Theorem 4.4).

In Section 5 the masking function is supposed to be known only on an open ring around the trace of a multicurve. First, it is shown that multisegments can be distinguished from each other by this restricted knowledge (Theorem 5.1). Then it is proved that if a countable set of masking functions of the multicurve are given on that open ring around the trace so that the d-weights establish a complete system of independent functions, then the multicurve can be recovered (Theorem 5.2).

Section 6 is devoted to the proof of our Conjecture for constant weight (as it was raised in [10, Conjecture]), but with the assumption that the multicurve is real analytic with non-vanishing curvature (see Theorem 6.2).

Finally, Section 7 provides some possible generalizations to higher dimensions, raises questions for further investigation, and discusses some consequences of forgoing results. One of these is Corollary 7.1, a statement similar in spirit to J. C. C. Nitsche's result in [15], stating that only concentric multicircles can have a rotationally invariant masking function in a circular ring.

\section{Notations, terms and preliminaries}

We use the notation $f\left(t^{ \pm}\right):=f(t \pm)=\lim _{0<\varepsilon \rightarrow 0} f(t \pm \varepsilon)$ for any real function $f$, and the short-hands $\boldsymbol{u}_{\beta}=(\cos \beta, \sin \beta)$, and $\boldsymbol{u}_{X}(Y):=\frac{Y-X}{|Y-X|}$, the unit vector directing from the point $X$ toward the different point $Y$.

The linear map $\cdot^{\perp}$ on unit vectors is defined by $\boldsymbol{u}_{\beta}^{\perp}=\boldsymbol{u}_{\beta+\pi / 2}=(-\sin \beta, \cos \beta)$, and for general non-zero vectors by $\boldsymbol{x}^{\perp}=|\boldsymbol{x}|(\boldsymbol{x} /|\boldsymbol{x}|)^{\perp}$.

We denote the directional derivation by $\partial_{\boldsymbol{w}}$, where $\boldsymbol{w} \in \mathbb{S}^{1}$. Later we use the derivatives

$$
\begin{aligned}
g_{P}^{\boldsymbol{w}}(X) & :=\partial_{\boldsymbol{w}}\left(\frac{1}{|X-P|}\right)=\frac{-\langle\boldsymbol{w}, X-P\rangle}{|X-P|^{3}}=\frac{-\left\langle\boldsymbol{w}, \boldsymbol{u}_{P}(X)\right\rangle}{|X-P|^{2}}, \\
h_{P}^{\boldsymbol{w}}(X) & :=\partial_{\boldsymbol{w}}\left(\boldsymbol{u}_{P}(X)\right)=\partial_{\boldsymbol{w}}\left(\frac{X-P}{|X-P|}\right)=\frac{\boldsymbol{w}}{|X-P|}-(X-P) \frac{\left\langle\boldsymbol{w}, \boldsymbol{u}_{P}(X)\right\rangle}{|X-P|^{2}} \\
& =\frac{\boldsymbol{w}-\left\langle\boldsymbol{w}, \boldsymbol{u}_{P}(X)\right\rangle \boldsymbol{u}_{P}(X)}{|X-P|}=\frac{\left\langle\boldsymbol{w}, \boldsymbol{u}_{P}^{\perp}(X)\right\rangle}{|X-P|} \boldsymbol{u}_{P}^{\perp}(X),
\end{aligned}
$$

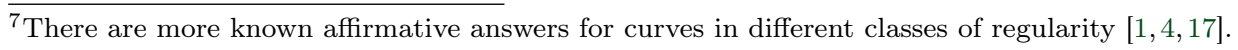


and

$$
\begin{aligned}
f_{P}^{\boldsymbol{w}}(X) & :=\partial_{\boldsymbol{w}}\left(\frac{\boldsymbol{u}_{P}(X)}{|X-P|}\right)=g_{P}^{\boldsymbol{w}}(X) \boldsymbol{u}_{P}(X)+\frac{h_{P}^{\boldsymbol{w}}(X)}{|X-P|} \\
& =\frac{-\left\langle\boldsymbol{w}, \boldsymbol{u}_{P}(X)\right\rangle}{|X-P|^{2}} \boldsymbol{u}_{P}(X)+\frac{\left\langle\boldsymbol{w}, \boldsymbol{u}_{P}^{\perp}(X)\right\rangle}{|X-P|^{2}} \boldsymbol{u}_{P}^{\perp}(X)=\frac{-1}{|X-P|^{2}} \tau_{P}^{X}(\boldsymbol{w}),
\end{aligned}
$$

where $\tau_{P}^{X}$ is the reflection with respect to the straight line $P X$.

Given a twice differentiable curve $\boldsymbol{r}$ in the plane with arc-length parameter $s$, we define its signed curvature by $\kappa^{e}(s)=\left\langle\ddot{\boldsymbol{r}}(s), \dot{\boldsymbol{r}}^{\perp}(s)\right\rangle$ and let $\kappa(s)=\left|\kappa^{e}(s)\right|$ be its curvature. We say that a non-degenerate segment of the form $\boldsymbol{r}\left(\left[s_{0}, s_{1}\right]\right)$ $\left(s_{0}<s_{1}\right)$ is traced. A traced segment $\boldsymbol{r}\left(\left[s_{0}, s_{1}\right]\right)$ is called maximal, if there is an $\varepsilon>0$ so that $\left[s_{0}, s_{1}\right]=\left[s_{0}^{\prime}, s_{1}^{\prime}\right]$ follows for any traced segment $\boldsymbol{r}\left(\left[s_{0}^{\prime}, s_{1}^{\prime}\right]\right)$ that satisfies $\left[s_{0}, s_{1}\right] \subseteq\left[s_{0}^{\prime}, s_{1}^{\prime}\right] \subseteq\left[s_{0}-\varepsilon, s_{1}+\varepsilon\right]$. We call also a straight line traced if there is a traced segment on it.

Let $\mathfrak{C}$ be the set of curves that

- are twice differentiable,

- are not self-intersecting,

- are parametrized by arc-length on a finite closed interval,

- are intersecting every straight line in only finitely many closed (maybe degenerate) segments,

- have only finitely many tangents through any point of its exterior,

- have only finitely many points of vanishing curvature beside a finite set of traced straight lines, and

- have only finitely many multiple tangent lines.

For any curve $\boldsymbol{r} \in \mathfrak{C}$ we introduce the notations

$\mathbb{R}_{\boldsymbol{r}}$ as the domain of the arc-length parameter $s$,

$\mathcal{L}_{\boldsymbol{r}}$ as the set of traced straight lines of $\boldsymbol{r}$,

$\Sigma_{\boldsymbol{r}}:=\left\{s \in \mathbb{R}_{\boldsymbol{r}}: \boldsymbol{r}(s)\right.$ is in a traced segment $\}$.

Given also a point $X$, we define

$\Theta_{\boldsymbol{r}}^{X}:=\left\{s \in \mathbb{R}_{\boldsymbol{r}}: X \in \ell(\boldsymbol{r}(s), \dot{\boldsymbol{r}}(s))\right\}, \tilde{\Theta}_{\boldsymbol{r}}^{X}:=\Theta_{\boldsymbol{r}}^{X} \backslash \Sigma_{\boldsymbol{r}}, \bar{\Theta}_{\boldsymbol{r}}^{X}:=\Theta_{\boldsymbol{r}}^{X} \cap \Sigma_{\boldsymbol{r}}$, and $\mathbb{R}_{\boldsymbol{r}}^{X}:=\mathbb{R}_{\boldsymbol{r}} \backslash \Theta_{\boldsymbol{r}}^{X}$.

Using the function $\sigma_{\boldsymbol{r}}^{X}(s):=\operatorname{sign}\left(\left\langle\dot{\boldsymbol{r}}(s), \boldsymbol{u}_{\boldsymbol{r}(s)}^{\perp}(X)\right\rangle\right)$ we see that

(a) $\sigma_{\boldsymbol{r}}^{X}(s)=0$ if and only if $s \in \Theta_{\boldsymbol{r}}^{X}$,

(b) if $s \in \Theta_{\boldsymbol{r}}^{X}$, then $s \in \bar{\Theta}_{\boldsymbol{r}}^{X}$ if and only if $\sigma_{\boldsymbol{r}}^{X}\left(s^{-}\right)=0$ or $\sigma_{\boldsymbol{r}}^{X}\left(s^{+}\right)=0$,

(c) if $s \in \tilde{\Theta}_{\boldsymbol{r}}^{X}$, then there is a neighbourhood of $\boldsymbol{r}(s)$ in which the curve $\boldsymbol{r}$ stays in one side of the straight line $\boldsymbol{r}(s) X$ if and only if $\sigma_{\boldsymbol{r}}^{X}\left(s^{-}\right) \neq \sigma_{\boldsymbol{r}}^{X}\left(s^{+}\right)$.

This enables us to define

$\hat{\Theta}_{\boldsymbol{r}}^{X}:=\left\{s \in \tilde{\Theta}_{\boldsymbol{r}}^{X}: \sigma_{\boldsymbol{r}}^{X}\left(s^{-}\right) \neq \sigma_{\boldsymbol{r}}^{X}\left(s^{+}\right)\right\}$. 


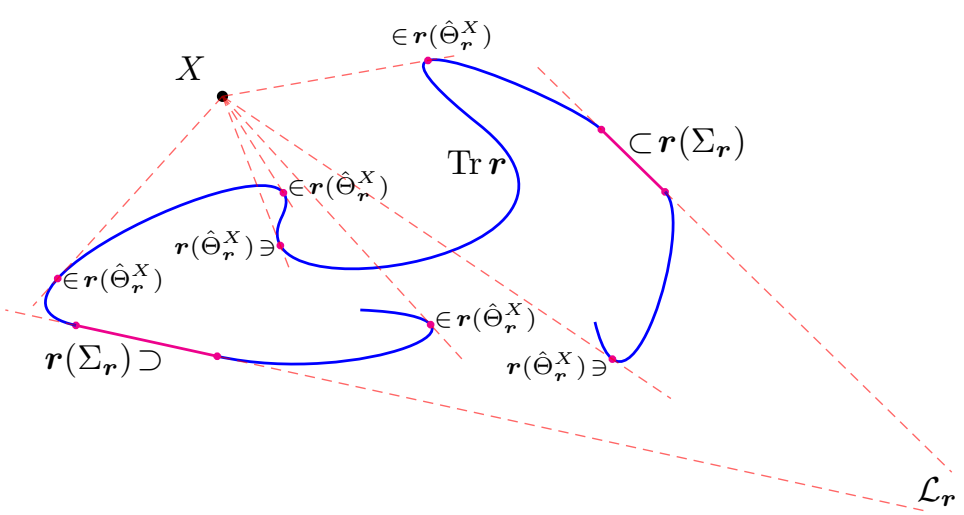

Figure 2.1. Points of $\boldsymbol{r}\left(\hat{\Theta}_{r}^{X}\right)$ and the traced lines in $\mathcal{L}_{\boldsymbol{r}}$

Further, let the set $\tilde{\mathfrak{C}}$ contain those curves of $\mathfrak{C}$ that

- have no non-degenerate segments in their traces, and let $\mathfrak{C}^{\mathcal{D}}$ and $\tilde{\mathfrak{C}}^{\mathcal{D}}$, respectively, contain those curves of $\mathfrak{C}$ and $\tilde{\mathfrak{C}}$ whose

- traces are in the compact domain $\mathcal{D} \subset \mathbb{R}^{2}$.

We also use these notations, properties and sets for multicurves, and to avoid long analytic technicalities ${ }^{8}$,

we confine ourselves to considering only multicurves in $\mathfrak{C}$.

Finally we note that a curve $r: \mathbb{R}_{\boldsymbol{r}} \rightarrow \mathbb{R}^{2}$ may be considered as a multicurve that has only one member, i.e. $\boldsymbol{r}$, but can also be regarded as a multicurve that has several parts $\boldsymbol{r}_{j}:=\left.\boldsymbol{r}\right|_{\left[a_{j}, a_{j+1}\right]}:\left[a_{j}, a_{j+1}\right] \rightarrow \mathbb{R}^{2}\left(\cdots<a_{j-1}<\cdots<a_{j}<a_{j+1}<\ldots\right.$, $j \in \mathcal{J} \subseteq \mathbb{N}$ ) of $\boldsymbol{r}_{\mathcal{J}}$ as members. Such parts of a curve are called subcurves, and we say in such a case that the curve is partitioned (into subcurves). A multicurve the members of which are exactly the subcurves of the members of another multicurve is also said to be the partition of that other multicurve.

We call a positive function $\omega: \mathbb{R}^{2} \backslash\{0\} \rightarrow \mathbb{R}$ of class $\mathrm{C}^{2}$ a directional weight, if it is homogeneous of degree 0, i.e., $\omega(\boldsymbol{x})=\omega(\lambda \boldsymbol{x})$ for any $\lambda \in \mathbb{R} \backslash\{0\}$. Notice that the derivative of such a function is

$$
\dot{\omega}(\boldsymbol{x})=\frac{\left\langle\dot{\omega}(\boldsymbol{x}), \boldsymbol{x}^{\perp}\right\rangle}{|\boldsymbol{x}|^{2}} \boldsymbol{x}^{\perp}
$$

because $0=\left.\frac{d}{d \lambda}\right|_{0}(\omega(\boldsymbol{x}+\lambda \boldsymbol{x}))=\langle\dot{\omega}(\boldsymbol{x}), \boldsymbol{x}\rangle$.

$\overline{{ }^{8} \text { Also ensures that the masking number }}$ is finite at every point. 
We define the d-weighted masking function $\bar{M}_{\boldsymbol{r} ; \omega}: \mathbb{R}^{2} \rightarrow \mathbb{R}$ of a multicurve $\boldsymbol{r}_{\mathcal{J}} \in \mathfrak{C}$ by

$$
\bar{M}_{\boldsymbol{r}_{\mathcal{J}} ; \omega}(X)=\int_{0}^{\pi} \omega\left(\boldsymbol{u}_{\alpha}\right) \#\left(\operatorname{Tr} \boldsymbol{r}_{\mathcal{J}} \cap \ell\left(X, \boldsymbol{u}_{\alpha}\right)\right) d \alpha
$$

where $\omega$ is a directional weight. The value $\bar{M}_{\boldsymbol{r}_{\mathcal{J}} ; \omega}(X)$ is called the $d$-weighted masking number of the multicurve $\boldsymbol{r}_{\mathcal{J}} \in \mathfrak{C}$ at $X \in \mathbb{R}^{2}$, while $\boldsymbol{r}_{\mathcal{J}} \in \mathfrak{C}$ is called the multicurve of the masking function $\bar{M}_{\boldsymbol{r}_{\mathcal{J}} ; \omega}$. We clearly have

$$
\bar{M}_{\boldsymbol{r}_{\mathcal{J}} ; \omega}(X)=\sum_{j \in \mathcal{J}} \bar{M}_{\boldsymbol{r}_{j} ; \omega}(X)
$$

and also $M_{\operatorname{Tr} \boldsymbol{r}_{\mathcal{J}} ; \bar{\omega}}=\bar{M}_{\boldsymbol{r}_{\mathcal{J}} ; \omega}$, because if $\omega$ is a directional weight, then $\bar{\omega}(\ell(P, \boldsymbol{w}))=$ $\omega(\boldsymbol{w})$ is a d-weight ${ }^{9}$ on $\mathcal{L}$.

Lemma 2.1. If $\boldsymbol{r} \in \tilde{\mathfrak{C}}$, then for any $X \in \mathbb{R}^{2}$, if $\boldsymbol{r} \in \mathfrak{C}$, then for $X \notin \operatorname{Tr} \boldsymbol{r}$, we have

$$
\bar{M}_{\boldsymbol{r} ; \omega}(X)=\int_{\mathbb{R}_{\boldsymbol{r}}} \omega(X-\boldsymbol{r}(s)) \frac{\left|\left\langle\dot{\boldsymbol{r}}(s), \boldsymbol{u}_{\boldsymbol{r}(s)}^{\perp}(X)\right\rangle\right|}{|X-\boldsymbol{r}(s)|} d s .
$$

Proof. Differentiation of the equation $\boldsymbol{u}_{\alpha}=\boldsymbol{u}_{X}(\boldsymbol{r}(s))$ with respect to $s$, if $X \neq \boldsymbol{r}(s)$, leads to

$$
\boldsymbol{u}_{\alpha}^{\perp} \frac{d \alpha}{d s}=\frac{d \boldsymbol{u}_{\alpha}}{d s}=\frac{d}{d s}\left(\frac{\boldsymbol{r}(s)-X}{|\boldsymbol{r}(s)-X|}\right)=\frac{\dot{\boldsymbol{r}}(s)}{|\boldsymbol{r}(s)-X|}-\boldsymbol{u}_{X}(\boldsymbol{r}(s)) \frac{2\langle\dot{\boldsymbol{r}}(s), \boldsymbol{r}(s)-X\rangle)}{|\boldsymbol{r}(s)-X|},
$$

hence $d \alpha / d s=\left\langle\dot{\boldsymbol{r}}(s), \boldsymbol{u}_{X}^{\perp}(\boldsymbol{r}(s))\right\rangle /|\boldsymbol{r}(s)-X|$. If $X=\boldsymbol{r}\left(s_{0}\right)$, then

$$
\begin{aligned}
& \lim _{s \rightarrow s_{0}} \frac{\left\langle\dot{\boldsymbol{r}}(s), \boldsymbol{u}_{X}^{\perp}(\boldsymbol{r}(s))\right\rangle}{|\boldsymbol{r}(s)-X|} \\
& \quad=\lim _{s \rightarrow s_{0}} \frac{-\left\langle\left(s-s_{0}\right) \ddot{\boldsymbol{r}}^{\perp}\left(s_{0}\right)+\dot{\boldsymbol{r}}^{\perp}\left(s_{0}\right),\left(s-s_{0}\right) \dot{\boldsymbol{r}}\left(s_{0}\right)+\frac{1}{2}\left(s-s_{0}\right)^{2} \ddot{\boldsymbol{r}}\left(s_{0}\right)\right\rangle}{\left|s-s_{0}\right|^{2}}=\frac{\kappa^{e}\left(s_{0}\right)}{2},
\end{aligned}
$$

therefore changing the variable $\boldsymbol{u}_{\alpha}=\boldsymbol{u}_{X}(\boldsymbol{r}(s))$ in (2.3) implies the lemma.

Notice that the integration in the lemma could be restricted to $\mathbb{R}_{\boldsymbol{r}}^{X}$, as the integrand vanishes on $\Theta_{r}^{X}$.

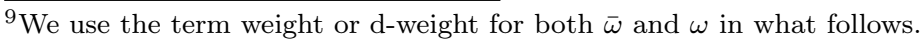




\section{Derivatives of the d-weighted masking function}

Now, we are ready to differentiate the masking function.

Proposition 3.1. If $\boldsymbol{r} \in \tilde{\mathfrak{C}}$, then for any $X \in \mathbb{R}^{2}$, if $\boldsymbol{r} \in \mathfrak{C}$, then for $X \notin \operatorname{Tr} \boldsymbol{r}$, we have

$$
\begin{aligned}
\partial_{\boldsymbol{w}} \bar{M}_{\boldsymbol{r} ; \omega}(X)= & \int_{\mathbb{R}_{\boldsymbol{r}}^{X}} \omega(X-\boldsymbol{r}(s)) \frac{\left\langle\tau_{X}^{\boldsymbol{r}(s)}(\dot{\boldsymbol{r}}(s)), \boldsymbol{w}^{\perp}\right\rangle}{\sigma_{\boldsymbol{r}}^{X}(s)|X-\boldsymbol{r}(s)|^{2}} d s+ \\
& +\int_{\mathbb{R}_{\boldsymbol{r}}^{X}}\langle\dot{\omega}(X-\boldsymbol{r}(s)), \boldsymbol{w}\rangle \frac{\left\langle\dot{\boldsymbol{r}}(s), \boldsymbol{u}_{\boldsymbol{r}(s)}^{\perp}(X)\right\rangle}{\sigma_{\boldsymbol{r}}^{X}(s)|X-\boldsymbol{r}(s)|} d s,
\end{aligned}
$$

where $\boldsymbol{w} \in \mathbb{S}^{1}$ and $\sigma_{\boldsymbol{r}}^{X}(s)=\operatorname{sign}\left(\left\langle\dot{\boldsymbol{r}}(s), \boldsymbol{u}_{\boldsymbol{r}(s)}^{\perp}(X)\right\rangle\right)$.

Proof. Lemma 2.1 clearly implies

$$
\partial_{\boldsymbol{w}} \bar{M}_{\boldsymbol{r} ; \omega}(X)=\int_{\mathbb{R}_{\boldsymbol{r}}} \partial_{\boldsymbol{w}}\left(\omega(X-\boldsymbol{r}(s)) \frac{\left\langle\dot{\boldsymbol{r}}(s), \boldsymbol{u}_{\boldsymbol{r}(s)}^{\perp}(X)\right\rangle}{\sigma_{\boldsymbol{r}}^{X}(s)|X-\boldsymbol{r}(s)|}\right) d s
$$

which leads to

$$
\begin{aligned}
& \partial_{\boldsymbol{w}} \bar{M}_{\boldsymbol{r} ; \omega}(X) \\
& =\int_{\mathbb{R}_{\boldsymbol{r}}^{X}} \omega(X-\boldsymbol{r}(s))\left(g_{\boldsymbol{r}(s)}^{\boldsymbol{w}}(X) \frac{\left\langle\dot{\boldsymbol{r}}(s), \boldsymbol{u}_{\boldsymbol{r}(s)}^{\perp}(X)\right\rangle}{\sigma_{\boldsymbol{r}}^{X}(s)}+\frac{\left\langle\dot{\boldsymbol{r}}(s),\left(h_{\boldsymbol{r}(s)}^{\boldsymbol{w}}(X)\right)^{\perp}\right\rangle}{\sigma_{\boldsymbol{r}}^{X}(s)|X-\boldsymbol{r}(s)|}\right) d s+ \\
& \quad+\int_{\mathbb{R}_{\boldsymbol{r}}^{X}}\langle\dot{\omega}(X-\boldsymbol{r}(s)), \boldsymbol{w}\rangle \frac{\left\langle\dot{\boldsymbol{r}}(s), \boldsymbol{u}_{\boldsymbol{r}(s)}^{\perp}(X)\right\rangle}{\sigma_{\boldsymbol{r}}^{X}(s)|X-\boldsymbol{r}(s)|} d s .
\end{aligned}
$$

The second integral above is the second member of the stated formula, so we only need to calculate the first integral. Substituting our expressions for $g^{\boldsymbol{w}}$ and $h^{\boldsymbol{w}}$ into the first integral we obtain

$$
\begin{aligned}
\int_{\mathbb{R}_{\boldsymbol{r}}^{X}} \omega(X & -\boldsymbol{r}(s)) \times \\
& \times\left(\frac{-\left\langle\boldsymbol{w}, \boldsymbol{u}_{\boldsymbol{r}(s)}(X)\right\rangle\left\langle\dot{\boldsymbol{r}}(s), \boldsymbol{u}_{\boldsymbol{r}(s)}^{\perp}(X)\right\rangle}{\sigma_{\boldsymbol{r}}^{X}(s)|X-\boldsymbol{r}(s)|^{2}}-\frac{\left\langle\dot{\boldsymbol{r}}(s),\left\langle\boldsymbol{w}, \boldsymbol{u}_{\boldsymbol{r}(s)}^{\perp}(X)\right\rangle \boldsymbol{u}_{\boldsymbol{r}(s)}(X)\right\rangle}{\sigma_{\boldsymbol{r}}^{X}(s)|X-\boldsymbol{r}(s)|^{2}}\right) d s \\
= & \int_{\mathbb{R}_{\boldsymbol{r}}^{X}} \omega(X-\boldsymbol{r}(s)) \times \\
& \times \frac{\left\langle\boldsymbol{w}^{\perp},-\left\langle\dot{\boldsymbol{r}}(s), \boldsymbol{u}_{\boldsymbol{r}(s)}^{\perp}(X)\right\rangle \boldsymbol{u}_{\boldsymbol{r}(s)}^{\perp}(X)+\left\langle\dot{\boldsymbol{r}}(s), \boldsymbol{u}_{\boldsymbol{r}(s)}(X)\right\rangle \boldsymbol{u}_{\boldsymbol{r}(s)}(X)\right\rangle}{\sigma_{\boldsymbol{r}}^{X}(s)|\boldsymbol{r}(s)-X|^{2}} d s
\end{aligned}
$$

which completes the proof. 
If the curve is a segment, then differentiation leads to the following.

Proposition 3.2. If $\boldsymbol{r}:[0, h] \ni s \mapsto \boldsymbol{r}(0)+s \boldsymbol{v}\left(\boldsymbol{v} \in \mathbb{S}^{1}\right)$, then

$$
\begin{aligned}
\partial_{\boldsymbol{w}} & \bar{M}_{\boldsymbol{r} ; \omega}(X) \\
\quad & = \begin{cases}-\omega(\boldsymbol{v})\left|\left\langle\boldsymbol{v}, \boldsymbol{w}^{\perp}\right\rangle\right|\left(\frac{1}{x}+\frac{1}{h-x}\right), & \text { if } X=\boldsymbol{r}(0)+x \boldsymbol{v} \text { and } x \in \mathbb{R} \backslash\{0, h\}, \\
-\partial_{-\boldsymbol{w}} \bar{M}_{\boldsymbol{r} ; \omega}(X), & \text { if } X \notin \ell(\boldsymbol{r}(0), \boldsymbol{v}) .\end{cases}
\end{aligned}
$$

Proof. The second case of formula (3.1) follows directly from Proposition 3.1, so from now on, we assume $X \in \ell(\boldsymbol{r}(0), \boldsymbol{v})$, i.e. $X=\boldsymbol{r}(0)+x \boldsymbol{v}(x \in \mathbb{R})$, and consider only the first case.

Observe that

$$
2 \bar{M}_{\boldsymbol{r} ; \omega}(X)= \begin{cases}\int_{-\pi}^{\pi} \omega\left(\boldsymbol{u}_{\alpha}\right) d \alpha, & \text { if } X \in \operatorname{Tr} \boldsymbol{r} \\ 0, & \text { if } X \in \ell(\boldsymbol{r}(0), \boldsymbol{v}) \backslash \operatorname{Tr} \boldsymbol{r} .\end{cases}
$$

This immediately gives $\partial_{\boldsymbol{v}} \bar{M}_{\boldsymbol{r} ; \omega}(X)=0$ if $x \neq h$ and $\partial_{-\boldsymbol{v}} \bar{M}_{\boldsymbol{r} ; \omega}(X)=0$ if $x \neq 0$.

Now, we assume that $\boldsymbol{w}$ is not parallel to $\boldsymbol{v}$. Then we have an $\varepsilon>0$ so that $X+t \boldsymbol{w} \notin \operatorname{Tr} \boldsymbol{r}$ for every $t \in(0, \varepsilon)$, hence Lemma 2.1 gives

$$
\begin{aligned}
\bar{M}_{\boldsymbol{r} ; \omega}(X+t \boldsymbol{w}) & =\int_{0}^{h} \omega(X+t \boldsymbol{w}-\boldsymbol{r}(s)) \frac{\left|\left\langle\boldsymbol{v}, \boldsymbol{u}_{\boldsymbol{r}(s)}^{\perp}(X+t \boldsymbol{w})\right\rangle\right|}{|X+t \boldsymbol{w}-\boldsymbol{r}(s)|} d s \\
& =\int_{0}^{h} \omega((x-s) \boldsymbol{v}+t \boldsymbol{w}) \frac{\left|\left\langle\boldsymbol{v}^{\perp},(x-s) \boldsymbol{v}+t \boldsymbol{w}\right\rangle\right|}{|(x-s) \boldsymbol{v}+t \boldsymbol{w}|^{2}} d s \\
& =t\left|\left\langle\boldsymbol{v}^{\perp}, \boldsymbol{w}\right\rangle\right| \int_{0}^{h} \frac{\omega((x-s) \boldsymbol{v}+t \boldsymbol{w})}{|(x-s) \boldsymbol{v}+t \boldsymbol{w}|^{2}} d s .
\end{aligned}
$$

If $X \in \operatorname{Tr} \boldsymbol{r}$, then substituting $\boldsymbol{u}_{\alpha}=\frac{y \boldsymbol{v}+t \boldsymbol{w}}{|y \boldsymbol{v}+t \boldsymbol{w}|}$ into (3.2) leads to

$$
\begin{aligned}
\bar{M}_{\boldsymbol{r} ; \omega}(X) & =\int_{-\infty}^{\infty} \omega(y \boldsymbol{v}+t \boldsymbol{w})\left|\frac{d \alpha}{d y}\right| d y=\int_{-\infty}^{\infty} \omega(y \boldsymbol{v}+t \boldsymbol{w}) \frac{t\left|\left\langle\boldsymbol{v}^{\perp}, \boldsymbol{w}\right\rangle\right|}{|y \boldsymbol{v}+t \boldsymbol{w}|^{2}} d y \\
& =t\left|\left\langle\boldsymbol{v}^{\perp}, \boldsymbol{w}\right\rangle\right| \int_{-\infty}^{\infty} \frac{\omega((x-s) \boldsymbol{v}+t \boldsymbol{w})}{|(x-s) \boldsymbol{v}+t \boldsymbol{w}|^{2}} d s
\end{aligned}
$$


hence (3.3) gives

$$
\begin{aligned}
\partial_{\boldsymbol{w}} & \bar{M}_{\boldsymbol{r} ; \omega}(X) \\
& =\lim _{t \searrow 0} \frac{\bar{M}_{\boldsymbol{r} ; \omega}(X+t \boldsymbol{w})-\bar{M}_{\boldsymbol{r} ; \omega}(X)}{t} \\
& =-\left|\left\langle\boldsymbol{v}^{\perp}, \boldsymbol{w}\right\rangle\right| \lim _{t \searrow 0}\left(\int_{-\infty}^{0} \frac{\omega((x-s) \boldsymbol{v}+t \boldsymbol{w})}{|(x-s) \boldsymbol{v}+t \boldsymbol{w}|^{2}} d s+\int_{h}^{\infty} \frac{\omega((x-s) \boldsymbol{v}+t \boldsymbol{w})}{|(x-s) \boldsymbol{v}+t \boldsymbol{w}|^{2}} d s\right) \\
& =-\left|\left\langle\boldsymbol{v}^{\perp}, \boldsymbol{w}\right\rangle\right| \omega(\boldsymbol{v})\left(\int_{-\infty}^{0} \frac{1}{(x-s)^{2}} d s+\int_{h}^{\infty} \frac{1}{(x-s)^{2}} d s\right) \\
& =-\left|\left\langle\boldsymbol{v}^{\perp}, \boldsymbol{w}\right\rangle\right| \omega(\boldsymbol{v})\left(\frac{1}{x}+\frac{1}{h-x}\right) .
\end{aligned}
$$

If $X \in \ell(\boldsymbol{r}(0), \boldsymbol{v}) \backslash \operatorname{Tr} \boldsymbol{r}$, then $\bar{M}_{\boldsymbol{r} ; \omega}(X)=0$ by (3.2), hence (3.3) gives

$$
\begin{aligned}
\partial_{\boldsymbol{w}} \bar{M}_{\boldsymbol{r} ; \omega}(X) & =\lim _{t \searrow 0} \frac{\bar{M}_{\boldsymbol{r} ; \omega}(X+t \boldsymbol{w})-\bar{M}_{\boldsymbol{r} ; \omega}(X)}{t} \\
& =\left|\left\langle\boldsymbol{v}^{\perp}, \boldsymbol{w}\right\rangle\right| \omega(\boldsymbol{v}) \int_{0}^{h} \frac{1}{(x-s)^{2}} d s=-\left|\left\langle\boldsymbol{v}^{\perp}, \boldsymbol{w}\right\rangle\right| \omega(\boldsymbol{v})\left(\frac{1}{x}+\frac{1}{h-x}\right) .
\end{aligned}
$$

The proof is complete.

Propositions 3.1 and 3.2 generalize and refine [8, Theorem 2].

The next theorem generalizes the first statement in [10, Lemma 1] and the displayed formula at [8, p. 371].

Theorem 3.3. For any curve $\boldsymbol{r} \in \mathfrak{C}$ that has $\Theta_{\boldsymbol{r}}^{X}=\left\{s_{i}\right\}_{i=1}^{m} \subset \mathbb{R}_{\boldsymbol{r}}=\left[s_{0}, s_{m+1}\right]$ for an $m \in \mathbb{N}$, where the sequence $s_{i}$ is strictly monotonously increasing, we have

$$
\begin{aligned}
\partial_{\boldsymbol{w}} \bar{M}_{\boldsymbol{r} ; \omega}(X)=2 \sigma_{\boldsymbol{r}}^{X}\left(s_{1}^{+}\right) & \sum_{i=1}^{m}(-1)^{i} \omega\left(X-\boldsymbol{r}\left(s_{i}\right)\right) \frac{\left\langle\boldsymbol{w}^{\perp}, \boldsymbol{u}_{\boldsymbol{r}\left(s_{i}\right)}(X)\right\rangle}{\left|X-\boldsymbol{r}\left(s_{i}\right)\right|}- \\
& -\sigma_{\boldsymbol{r}}^{X}\left(s_{0}^{+}\right) \omega\left(X-\boldsymbol{r}\left(s_{0}\right)\right) \frac{\left\langle\boldsymbol{w}^{\perp}, \boldsymbol{u}_{\boldsymbol{r}\left(s_{0}\right)}(X)\right\rangle}{\left|X-\boldsymbol{r}\left(s_{0}\right)\right|}+ \\
& +\sigma_{\boldsymbol{r}}^{X}\left(s_{m}^{+}\right) \omega\left(X-\boldsymbol{r}\left(s_{m+1}\right)\right) \frac{\left\langle\boldsymbol{w}^{\perp}, \boldsymbol{u}_{\boldsymbol{r}\left(s_{m+1}\right)}(X)\right\rangle}{\left|X-\boldsymbol{r}\left(s_{m+1}\right)\right|} .
\end{aligned}
$$

Proof. By Proposition 3.1 we have

$$
\begin{aligned}
\partial_{\boldsymbol{w}} \bar{M}_{\boldsymbol{r} ; \omega}(X)=\int_{\mathbb{R}_{\boldsymbol{r}}} & \omega(X-\boldsymbol{r}(s)) \frac{\left\langle\tau_{X}^{\boldsymbol{r}(s)}(\dot{\boldsymbol{r}}(s)), \boldsymbol{w}^{\perp}\right\rangle}{\sigma_{\boldsymbol{r}}^{X}(s)|X-\boldsymbol{r}(s)|^{2}} d s- \\
& -\int_{\mathbb{R}_{\boldsymbol{r}}}\langle\dot{\omega}(X-\boldsymbol{r}(s)), \boldsymbol{w}\rangle \frac{\left\langle\dot{\boldsymbol{r}}(s), \boldsymbol{u}_{\boldsymbol{r}(s)}^{\perp}(X)\right\rangle}{\sigma_{\boldsymbol{r}}^{X}(s)|X-\boldsymbol{r}(s)|} d s=: I_{1}-I_{2} .
\end{aligned}
$$


As $\dot{\omega}(X-\boldsymbol{r}(s))=\left\langle\dot{\omega}(X-\boldsymbol{r}(s)), \boldsymbol{u}_{\boldsymbol{r}(s)}^{\perp}(X)\right\rangle \boldsymbol{u}_{\boldsymbol{r}(s)}^{\perp}(X)$ by $(2.2)$, we have

$$
\begin{aligned}
I_{2} & =\int_{\mathbb{R}_{\boldsymbol{r}}}\left\langle\dot{\omega}(X-\boldsymbol{r}(s)), \boldsymbol{u}_{\boldsymbol{r}(s)}^{\perp}(X)\right\rangle\left\langle\boldsymbol{u}_{\boldsymbol{r}(s)}^{\perp}(X), \boldsymbol{w}\right\rangle \frac{\left\langle\dot{\boldsymbol{r}}(s), \boldsymbol{u}_{\boldsymbol{r}(s)}^{\perp}(X)\right\rangle}{\sigma_{\boldsymbol{r}}^{X}(s)|X-\boldsymbol{r}(s)|} d s \\
& =-\int_{\mathbb{R}_{\boldsymbol{r}}} \frac{d}{d s}(\omega(X-\boldsymbol{r}(s))) \frac{\left\langle\boldsymbol{w}^{\perp}, \boldsymbol{u}_{\boldsymbol{r}(s)}(X)\right\rangle}{\sigma_{\boldsymbol{r}}^{X}(s)|X-\boldsymbol{r}(s)|} d s .
\end{aligned}
$$

Then, integration by parts leads to

$$
\begin{aligned}
I_{2}=\int_{\mathbb{R}_{\boldsymbol{r}}} \omega(X-\boldsymbol{r}(s)) & \frac{d}{d s}\left(\frac{\left\langle\boldsymbol{w}^{\perp}, \boldsymbol{u}_{\boldsymbol{r}(s)}(X)\right\rangle}{\sigma_{\boldsymbol{r}}^{X}(s)|X-\boldsymbol{r}(s)|}\right) d s- \\
& -\sum_{i=0}^{m} \sigma_{\boldsymbol{r}}^{X}\left(s_{i}^{+}\right)\left[\omega(X-\boldsymbol{r}(s)) \frac{\left\langle\boldsymbol{w}^{\perp}, \boldsymbol{u}_{\boldsymbol{r}(s)}(X)\right\rangle}{|X-\boldsymbol{r}(s)|}\right]_{s_{i}}^{s_{i+1}} .
\end{aligned}
$$

Using (2.1) the derivative in the integrand $\left(s \notin \hat{\Theta}_{r}^{X}\right)$ is

$$
\frac{d}{d s}\left(\frac{\left\langle\boldsymbol{w}^{\perp}, \boldsymbol{u}_{\boldsymbol{r}(s)}(X)\right\rangle}{\sigma_{\boldsymbol{r}}^{X}(s)|X-\boldsymbol{r}(s)|}\right)=\frac{-\left\langle\boldsymbol{w}^{\perp}, f_{X}^{\dot{\boldsymbol{r}}(s)}(\boldsymbol{r}(s))\right\rangle}{\sigma_{\boldsymbol{r}}^{X}(s)}=\frac{\left\langle\boldsymbol{w}^{\perp}, \tau_{X}^{\boldsymbol{r}(s)}(\dot{\boldsymbol{r}}(s))\right\rangle}{\sigma_{\boldsymbol{r}}^{X}(s)|X-\boldsymbol{r}(s)|^{2}}
$$

which eliminates $I_{1}$, hence we have

$$
\begin{aligned}
\partial_{\boldsymbol{w}} \bar{M}_{\boldsymbol{r} ; \omega}(X)= & \sum_{i=0}^{m} \sigma_{\boldsymbol{r}}^{X}\left(s_{i}^{+}\right)\left[\omega(X-\boldsymbol{r}(s)) \frac{\left\langle\boldsymbol{w}^{\perp}, \boldsymbol{u}_{\boldsymbol{r}(s)}(X)\right\rangle^{s_{i+1}}}{|\boldsymbol{r}(s)-X|}\right]_{s_{i}}^{s_{i}} \\
= & -\sigma_{\boldsymbol{r}}^{X}\left(s_{0}^{+}\right) \omega\left(X-\boldsymbol{r}\left(s_{0}\right)\right) \frac{\left\langle\boldsymbol{w}^{\perp}, \boldsymbol{u}_{\boldsymbol{r}\left(s_{0}\right)}(X)\right\rangle}{\left|\boldsymbol{r}\left(s_{0}\right)-X\right|}+ \\
& +\sum_{i=1}^{m}\left(\sigma_{X}^{\boldsymbol{r}}\left(s_{i-1}^{+}\right)-\sigma_{X}^{\boldsymbol{r}}\left(s_{i}^{+}\right)\right) \omega\left(X-\boldsymbol{r}\left(s_{i}\right)\right) \frac{\left\langle\boldsymbol{w}^{\perp}, \boldsymbol{u}_{\boldsymbol{r}\left(s_{i}\right)}(X)\right\rangle}{\left|\boldsymbol{r}\left(s_{i}\right)-X\right|}+ \\
& +\sigma_{\boldsymbol{r}}^{X}\left(s_{m}^{+}\right) \omega\left(X-\boldsymbol{r}\left(s_{m+1}\right)\right) \frac{\left\langle\boldsymbol{w}^{\perp}, \boldsymbol{u}_{\boldsymbol{r}\left(s_{m+1}\right)}(X)\right\rangle}{\left|\boldsymbol{r}\left(s_{m+1}\right)-X\right|}
\end{aligned}
$$

As $\sigma_{X}^{r}\left(s_{i-1}^{+}\right)-\sigma_{X}^{r}\left(s_{i}^{+}\right)=-2 \sigma_{X}^{r}\left(s_{i}^{+}\right)=2 \sigma_{X}^{r}\left(s_{1}^{+}\right)(-1)^{i}$, the theorem is proved.

The last two members of (3.4) disappear for closed curves, i.e. when $\boldsymbol{r}\left(s_{0}\right)=$ $\boldsymbol{r}\left(s_{m+1}\right)$, because $s_{0} \notin \hat{\Theta}_{\boldsymbol{r}}^{X}$ and $\sigma_{X}^{\boldsymbol{r}}\left(s_{m}^{+}\right)-\sigma_{X}^{\boldsymbol{r}}\left(s_{0}^{+}\right)=\sigma_{X}^{\boldsymbol{r}}\left(s_{m+1}^{-}\right)-\sigma_{X}^{\boldsymbol{r}}\left(s_{0}^{+}\right)=\sigma_{X}^{\boldsymbol{r}}\left(s_{0}^{-}\right)-$ $\sigma_{X}^{r}\left(s_{0}^{+}\right)=0$. Also note that the right-hand side of (3.4) does not really depend on $\sigma_{\boldsymbol{r}}^{X}\left(s_{1}^{+}\right)$, because $\sigma_{\boldsymbol{r}}^{X}\left(s_{1}^{+}\right)$is determined by the chosen direction of the curve's parametrization, and changing it to the opposite changes also the order of the points in $\hat{\Theta}_{\boldsymbol{r}}^{X}$ and $\sigma_{\boldsymbol{r}}^{X}\left(s_{1}^{+}\right)=\sigma_{\boldsymbol{r}}^{X}\left(s_{m}^{+}\right)(-1)^{m-1}=\sigma_{\boldsymbol{r}}^{X}\left(s_{m}^{-}\right)(-1)^{m}$.

Now, we generalize the second statement in [10, Lemma 1]. 
Theorem 3.4. Let $\boldsymbol{r} \in \mathfrak{C}$ and $\Theta_{\boldsymbol{r}}^{X}=\left\{s_{i}\right\}_{i=1}^{m} \subset \mathbb{R}_{r}=\left[s_{0}, s_{m+1}\right]$, where $s_{i}$ is strictly monotonously increasing $(m \in \mathbb{N})$. If $\kappa\left(s_{i}\right)>0$ for every $i=1, \ldots, m$, then

$$
\begin{aligned}
& \partial_{\boldsymbol{v}} \partial_{\boldsymbol{w}} \bar{M}_{\boldsymbol{r} ; \omega}(X) \\
& \begin{aligned}
=2 \sigma_{\boldsymbol{r}}^{X}\left(s_{1}^{+}\right) \sum_{i=1}^{m}(-1)^{i}\left[\frac{\left\langle\boldsymbol{w}^{\perp}, \boldsymbol{u}_{\boldsymbol{r}\left(s_{i}\right)}(X)\right\rangle}{\left|X-\boldsymbol{r}\left(s_{i}\right)\right|}\left\langle\dot{\omega}\left(X-\boldsymbol{r}\left(s_{i}\right)\right), \boldsymbol{v}\right\rangle-\right. \\
\quad-\frac{\omega\left(X-\boldsymbol{r}\left(s_{i}\right)\right)}{\left|X-\boldsymbol{r}\left(s_{i}\right)\right|^{2}}\left\langle\tau_{X}^{\boldsymbol{r}\left(s_{i}\right)}\left(\boldsymbol{w}^{\perp}\right), \boldsymbol{v}\right\rangle+ \\
\left.+\frac{\omega\left(X-\boldsymbol{r}\left(s_{i}\right)\right)}{\left|X-\boldsymbol{r}\left(s_{i}\right)\right|^{3} \kappa^{e}\left(s_{i}\right)}\left\langle\boldsymbol{w}^{\perp}, \boldsymbol{u}_{\boldsymbol{r}\left(s_{i}\right)}(X)\right\rangle\left\langle\dot{\boldsymbol{r}}^{\perp}\left(s_{i}\right), \boldsymbol{v}\right\rangle\right]- \\
-\sigma_{\boldsymbol{r}}^{X}\left(s_{0}^{+}\right)\left[\frac{\left\langle\boldsymbol{w}^{\perp}, \boldsymbol{u}_{\boldsymbol{r}\left(s_{0}\right)}(X)\right\rangle}{\left|X-\boldsymbol{r}\left(s_{0}\right)\right|}\left\langle\dot{\omega}\left(X-\boldsymbol{r}\left(s_{0}\right)\right), \boldsymbol{v}\right\rangle+\frac{\omega\left(X-\boldsymbol{r}\left(s_{0}\right)\right)}{\left|X-\boldsymbol{r}\left(s_{0}\right)\right|^{2}}\left\langle\tau_{X}^{\boldsymbol{r}\left(s_{0}\right)}\left(\boldsymbol{w}^{\perp}\right), \boldsymbol{v}\right\rangle\right]+ \\
+\sigma_{\boldsymbol{r}}^{X}\left(s_{m}^{+}\right)\left[\frac{\left\langle\boldsymbol{w}^{\perp}, \boldsymbol{u}_{\boldsymbol{r}\left(s_{m+1}\right)}(X)\right\rangle}{\left|X-\boldsymbol{r}\left(s_{m+1}\right)\right|}\left\langle\dot{\omega}\left(X-\boldsymbol{r}\left(s_{m+1}\right)\right), \boldsymbol{v}\right\rangle+\right. \\
\left.+\frac{\omega\left(X-\boldsymbol{r}\left(s_{m+1}\right)\right)}{\left|X-\boldsymbol{r}\left(s_{m+1}\right)\right|^{2}}\left\langle\tau_{X}^{\boldsymbol{r}\left(s_{m+1}\right)}\left(\boldsymbol{w}^{\perp}\right), \boldsymbol{v}\right\rangle\right] .
\end{aligned}
\end{aligned}
$$

Proof. Consider the parameters $s_{i}(i=1, \ldots, m)$ in (3.4) as functions $X \mapsto s_{i}(X)$ and take such an $\varepsilon>0$ that is small enough to have $X+t \boldsymbol{v} \in \ell\left(\boldsymbol{r}\left(s_{i}(X+t \boldsymbol{v}), \dot{\boldsymbol{r}}\left(s_{i}(X+\right.\right.\right.$ $t \boldsymbol{v})))$ for $t \in(0, \varepsilon)$. Then

$$
\begin{aligned}
\mid X+t \boldsymbol{v} & -\boldsymbol{r}\left(s_{i}(X+t \boldsymbol{v})\right) \mid \dot{\boldsymbol{r}}\left(s_{i}(X+t \boldsymbol{v})\right) \\
& =\left\langle\dot{\boldsymbol{r}}\left(s_{i}(X+t \boldsymbol{v})\right), \boldsymbol{u}_{\boldsymbol{r}\left(s_{i}(X+t \boldsymbol{v})\right)}(X+t \boldsymbol{v})\right\rangle\left(X+t \boldsymbol{v}-\boldsymbol{r}\left(s_{i}(X+t \boldsymbol{v})\right)\right),
\end{aligned}
$$

the right-derivative of which with respect to $t=0$ is

$$
\begin{aligned}
\left.\frac{d\left|X+t \boldsymbol{v}-\boldsymbol{r}\left(s_{i}(X+t \boldsymbol{v})\right)\right|}{d t}\right|_{t=0+} & \cdot \dot{\boldsymbol{r}}\left(s_{i}\right)+\left|X-\boldsymbol{r}\left(s_{i}\right)\right| \ddot{\boldsymbol{r}}\left(s_{i}\right) \partial_{\boldsymbol{v}} s_{i} \\
& =\left\langle\dot{\boldsymbol{r}}\left(s_{i} \pm_{i, \boldsymbol{v}}\right), \boldsymbol{u}_{\boldsymbol{r}\left(s_{i}\right)}(X)\right\rangle\left(\boldsymbol{v}-\dot{\boldsymbol{r}}\left(s_{i} \pm_{i, \boldsymbol{v}}\right) \partial_{\boldsymbol{v}} s_{i}\right),
\end{aligned}
$$

where $\pm_{i, \boldsymbol{v}}$ means just + or - depending on $i$ and $\boldsymbol{v}$. Multiplying this by $\dot{\boldsymbol{r}}^{\perp}\left(s_{i}\right)$ yields

$$
\frac{\left\langle\dot{\boldsymbol{r}}\left(s_{i}\right), \boldsymbol{u}_{\boldsymbol{r}\left(s_{i}\right)}(X)\right\rangle\left\langle\dot{\boldsymbol{r}}^{\perp}\left(s_{i}\right), \boldsymbol{v}\right\rangle}{\left|X-\boldsymbol{r}\left(s_{i}\right)\right|}=\left\langle\dot{\boldsymbol{r}}^{\perp}\left(s_{i}\right), \ddot{\boldsymbol{r}}\left(s_{i}\right)\right\rangle \partial_{\boldsymbol{v}} s_{i}=\kappa^{e}\left(s_{i}\right) \partial_{\boldsymbol{v}} s_{i} .
$$

Having this we differentiate (3.4) member by member and get

$$
\partial_{\boldsymbol{v}}\left(\omega\left(X-\boldsymbol{r}\left(s_{i}\right)\right) \frac{\left\langle\boldsymbol{w}^{\perp}, \boldsymbol{u}_{\boldsymbol{r}\left(s_{i}\right)}(X)\right\rangle}{\left|X-\boldsymbol{r}\left(s_{i}\right)\right|}\right)
$$




$$
\begin{aligned}
= & \left\langle\dot{\omega}\left(X-\boldsymbol{r}\left(s_{i}\right)\right), \boldsymbol{v}-\partial_{\boldsymbol{v}}\left(\boldsymbol{r}\left(s_{i}\right)\right)\right\rangle \frac{\left\langle\boldsymbol{w}^{\perp}, \boldsymbol{u}_{\boldsymbol{r}\left(s_{i}\right)}(X)\right\rangle}{\left|X-\boldsymbol{r}\left(s_{i}\right)\right|}+ \\
& +\omega\left(X-\boldsymbol{r}\left(s_{i}\right)\right) \frac{\left\langle\boldsymbol{w}^{\perp}, \boldsymbol{v}-\partial_{\boldsymbol{v}}\left(\boldsymbol{r}\left(s_{i}\right)\right)\right\rangle}{\left|X-\boldsymbol{r}\left(s_{i}\right)\right|^{2}}- \\
& -2 \omega\left(X-\boldsymbol{r}\left(s_{i}\right)\right) \frac{\left\langle\boldsymbol{w}^{\perp}, X-\boldsymbol{r}\left(s_{i}\right)\right\rangle\left\langle\boldsymbol{v}-\partial_{\boldsymbol{v}}\left(\boldsymbol{r}\left(s_{i}\right)\right), X-\boldsymbol{r}\left(s_{i}\right)\right\rangle}{\left|X-\boldsymbol{r}\left(s_{i}\right)\right|^{4}} \\
= & \left\langle\dot{\omega}\left(X-\boldsymbol{r}\left(s_{i}\right)\right), \boldsymbol{v}-\partial_{\boldsymbol{v}}\left(\boldsymbol{r}\left(s_{i}\right)\right)\right\rangle \frac{\left\langle\boldsymbol{w}^{\perp}, \boldsymbol{u}_{\boldsymbol{r}\left(s_{i}\right)}(X)\right\rangle}{\left|X-\boldsymbol{r}\left(s_{i}\right)\right|}+ \\
& +\frac{\omega\left(X-\boldsymbol{r}\left(s_{i}\right)\right)}{\left|X-\boldsymbol{r}\left(s_{i}\right)\right|^{2}}\left\langle\boldsymbol{w}^{\perp}-2\left\langle\boldsymbol{w}^{\perp}, \boldsymbol{u}_{\boldsymbol{r}\left(s_{i}\right)}(X)\right\rangle \boldsymbol{u}_{\boldsymbol{r}\left(s_{i}\right)}(X), \boldsymbol{v}-\partial_{\boldsymbol{v}}\left(\boldsymbol{r}\left(s_{i}\right)\right)\right\rangle \\
= & \frac{\left\langle\boldsymbol{w}^{\perp}, \boldsymbol{u} \boldsymbol{r}\left(s_{i}\right)\right.}{\left|X-\boldsymbol{r}\left(s_{i}\right)\right|}\left\langle\dot{\omega}\left(X-\boldsymbol{r}\left(s_{i}\right)\right), \boldsymbol{v}-\partial_{\boldsymbol{v}}\left(\boldsymbol{r}\left(s_{i}\right)\right)\right\rangle- \\
& \quad \frac{\omega\left(X-\boldsymbol{r}\left(s_{i}\right)\right)}{\left|X-\boldsymbol{r}\left(s_{i}\right)\right|^{2}}\left\langle\tau_{\boldsymbol{r}\left(s_{i}\right)}^{X}\left(\boldsymbol{w}^{\perp}\right), \boldsymbol{v}-\partial_{\boldsymbol{v}}\left(\boldsymbol{r}\left(s_{i}\right)\right)\right\rangle .
\end{aligned}
$$

This gives the last two members in the formula stated in the theorem as the endpoints of $\boldsymbol{r}$ are not in $\hat{\Theta}_{\boldsymbol{r}}^{X}$, and therefore $\partial_{\boldsymbol{v}}\left(\boldsymbol{r}\left(s_{0}\right)\right)=0=\partial_{\boldsymbol{v}}\left(\boldsymbol{r}\left(s_{m+1}\right)\right)$.

For the other members in the formula stated in the theorem we have $\partial_{\boldsymbol{v}}\left(\boldsymbol{r}\left(s_{i}\right)\right)=\dot{\boldsymbol{r}}\left(s_{i}\right) \partial_{\boldsymbol{v}} s_{i}$, hence by $(2.2),(3.5)$ and $\dot{\boldsymbol{r}}\left(s_{i}\right)=\left\langle\dot{\boldsymbol{r}}\left(s_{i}\right), \boldsymbol{u}_{\boldsymbol{r}\left(s_{i}\right)}(X)\right\rangle \boldsymbol{u}_{\boldsymbol{r}\left(s_{i}\right)}(X)$ yield

$$
\begin{aligned}
- & \frac{\left\langle\boldsymbol{w}^{\perp}, \boldsymbol{u}_{\boldsymbol{r}\left(s_{i}\right)}(X)\right\rangle}{\left|X-\boldsymbol{r}\left(s_{i}\right)\right|}\left\langle\dot{\omega}\left(X-\boldsymbol{r}\left(s_{i}\right)\right), \partial_{\boldsymbol{v}}\left(\boldsymbol{r}\left(s_{i}\right)\right)\right\rangle+\frac{\omega\left(X-\boldsymbol{r}\left(s_{i}\right)\right)}{\left|X-\boldsymbol{r}\left(s_{i}\right)\right|^{2}}\left\langle\tau_{X}^{\boldsymbol{r}\left(s_{i}\right)}\left(\boldsymbol{w}^{\perp}\right), \partial_{\boldsymbol{v}}\left(\boldsymbol{r}\left(s_{i}\right)\right)\right\rangle \\
= & \frac{\left\langle\boldsymbol{w}^{\perp}, \boldsymbol{u}_{\boldsymbol{r}\left(s_{i}\right)}(X)\right\rangle}{\left|X-\boldsymbol{r}\left(s_{i}\right)\right|}\left\langle\dot{\omega}\left(X-\boldsymbol{r}\left(s_{i}\right)\right), \dot{\boldsymbol{r}}\left(s_{i}\right) \partial_{\boldsymbol{v}} s_{i}\right\rangle+\frac{\omega\left(X-\boldsymbol{r}\left(s_{i}\right)\right)}{\left|X-\boldsymbol{r}\left(s_{i}\right)\right|^{2}}\left\langle\tau_{X}^{\boldsymbol{r}\left(s_{i}\right)}\left(\boldsymbol{w}^{\perp}\right), \dot{\boldsymbol{r}}\left(s_{i}\right) \partial_{\boldsymbol{v}} s_{i}\right\rangle \\
= & \frac{\omega\left(X-\boldsymbol{r}\left(s_{i}\right)\right)}{\left|X-\boldsymbol{r}\left(s_{i}\right)\right|^{2}}\left\langle\tau_{X}^{\boldsymbol{r}\left(s_{i}\right)}\left(\boldsymbol{w}^{\perp}\right), \dot{\boldsymbol{r}}\left(s_{i}\right) \frac{\left\langle\dot{\boldsymbol{r}}\left(s_{i}\right), \boldsymbol{u}_{\boldsymbol{r}\left(s_{i}\right)}(X)\right\rangle\left\langle\dot{\boldsymbol{r}}^{\perp}\left(s_{i}\right), \boldsymbol{v}\right\rangle}{\kappa^{e}\left(s_{i}\right)\left|X-\boldsymbol{r}\left(s_{i}\right)\right|}\right\rangle \\
= & \frac{\omega\left(X-\boldsymbol{r}\left(s_{i}\right)\right)}{\kappa^{e}\left(s_{i}\right)\left|X-\boldsymbol{r}\left(s_{i}\right)\right|^{3}}\left\langle\boldsymbol{w}^{\perp}, \boldsymbol{u}_{\boldsymbol{r}\left(s_{i}\right)}(X)\right\rangle\left\langle\dot{\boldsymbol{r}}^{\perp}\left(s_{i}\right), \boldsymbol{v}\right\rangle
\end{aligned}
$$

which completes the proof of the theorem.

Now we are able to determine the Laplacian $\Delta=\partial_{\boldsymbol{w}}^{2}+\partial_{\boldsymbol{w}^{\perp}}^{2}$ of the weighted masking function.

Theorem 3.5. Let $\boldsymbol{r} \in \mathfrak{C}$ and $\Theta_{\boldsymbol{r}}^{X}=\left\{s_{i}\right\}_{i=1}^{m} \subset \mathbb{R}_{r}=\left[s_{0}, s_{m+1}\right]$, where $s_{i}$ is strictly 
monotonously increasing $(m \in \mathbb{N})$. If $\kappa\left(s_{i}\right)>0$ for every $i=1, \ldots, m$, then

$$
\begin{aligned}
\Delta \bar{M}_{\boldsymbol{r} ; \omega}(X)= & \sigma_{\boldsymbol{r}}^{X}\left(s_{1}^{+}\right) \sum_{i=1}^{m}(-1)^{i}\left[\frac{\left\langle\dot{\omega}\left(X-\boldsymbol{r}\left(s_{i}\right)\right), \boldsymbol{u}_{\boldsymbol{r}\left(s_{i}\right)}^{\perp}(X)\right\rangle}{\left|X-\boldsymbol{r}\left(s_{i}\right)\right|}-\right. \\
& \left.-\frac{\omega\left(X-\boldsymbol{r}\left(s_{i}\right)\right)}{\left|X-\boldsymbol{r}\left(s_{i}\right)\right|^{3}} \frac{\left\langle\boldsymbol{u}_{\boldsymbol{r}\left(s_{i}\right)}(X), \dot{\boldsymbol{r}}\left(s_{i}\right)\right\rangle}{\kappa^{e}\left(s_{i}\right)}\right]+ \\
& +\sigma_{\boldsymbol{r}}^{X}\left(s_{0}^{+}\right) \frac{\left\langle\dot{\omega}\left(X-\boldsymbol{r}\left(s_{0}\right)\right), \boldsymbol{u}_{\boldsymbol{r}\left(s_{0}\right)}^{\perp}(X)\right\rangle}{\left|X-\boldsymbol{r}\left(s_{0}\right)\right|}- \\
& -\sigma_{\boldsymbol{r}}^{X}\left(s_{m}^{+}\right) \frac{\left\langle\dot{\omega}\left(X-\boldsymbol{r}\left(s_{m+1}\right)\right), \boldsymbol{u}_{\boldsymbol{r}\left(s_{m+1}\right)}^{\perp}(X)\right\rangle}{\left|X-\boldsymbol{r}\left(s_{m+1}\right)\right|}
\end{aligned}
$$

Proof. By the formula in Theorem 3.4 and $(2.2)$, we see that $\partial_{\boldsymbol{w}}^{2} \bar{M}_{\boldsymbol{r} ; \omega}(X)=$ $\partial_{\boldsymbol{w}} \partial_{\boldsymbol{w}} \bar{M}_{\boldsymbol{r} ; \omega}(X)$ is the alternating sum of the members

$$
\begin{aligned}
& \frac{\left\langle\dot{\omega}\left(X-\boldsymbol{r}\left(s_{i}\right)\right), \boldsymbol{u}_{\boldsymbol{r}\left(s_{i}\right)}^{\perp}(X)\right\rangle}{\left|X-\boldsymbol{r}\left(s_{i}\right)\right|}\left\langle\boldsymbol{w}^{\perp}, \boldsymbol{u}_{\boldsymbol{r}\left(s_{i}\right)}(X)\right\rangle\left\langle\boldsymbol{u}_{\boldsymbol{r}\left(s_{i}\right)}^{\perp}(X), \boldsymbol{w}\right\rangle- \\
& -\frac{\omega\left(X-\boldsymbol{r}\left(s_{i}\right)\right)}{\left|X-\boldsymbol{r}\left(s_{i}\right)\right|^{2}}\left\langle\tau_{X}^{\boldsymbol{r}\left(s_{i}\right)}\left(\boldsymbol{w}^{\perp}\right), \boldsymbol{w}\right\rangle+ \\
& +\frac{\omega\left(X-\boldsymbol{r}\left(s_{i}\right)\right)}{\left|X-\boldsymbol{r}\left(s_{i}\right)\right|^{3} \kappa^{e}\left(s_{i}\right)}\left\langle\boldsymbol{w}^{\perp}, \boldsymbol{u}_{\boldsymbol{r}\left(s_{i}\right)}(X)\right\rangle\left\langle\dot{\boldsymbol{r}}^{\perp}\left(s_{i}\right), \boldsymbol{w}\right\rangle .
\end{aligned}
$$

Since

$$
\begin{aligned}
\left\langle\boldsymbol{w}^{\perp}, \boldsymbol{u}_{\boldsymbol{r}\left(s_{i}\right)}(X)\right\rangle\left\langle\boldsymbol{u}_{\boldsymbol{r}\left(s_{i}\right)}^{\perp}(X), \boldsymbol{w}\right\rangle & =-\left\langle\boldsymbol{w}^{\perp}, \boldsymbol{u}_{\boldsymbol{r}\left(s_{i}\right)}(X)\right\rangle^{2}, \\
\left\langle\boldsymbol{w}^{\perp}, \boldsymbol{u}_{\boldsymbol{r}\left(s_{i}\right)}(X)\right\rangle^{2}+\left\langle\boldsymbol{w}, \boldsymbol{u}_{\boldsymbol{r}\left(s_{i}\right)}(X)\right\rangle^{2} & =1,
\end{aligned}
$$

and $\left\langle\tau_{X}^{\boldsymbol{r}\left(s_{i}\right)}\left(\boldsymbol{w}^{\perp}\right), \boldsymbol{w}\right\rangle=\left\langle\tau_{X}^{\boldsymbol{r}\left(s_{i}\right)}(\boldsymbol{w}), \boldsymbol{w}^{\perp}\right\rangle$, we obtain that

$$
\begin{aligned}
\partial_{\boldsymbol{w}}^{2} \bar{M}_{\boldsymbol{r} ; \omega}(X)+\partial_{\boldsymbol{w}^{\perp}}^{2} \bar{M}_{\boldsymbol{r} ; \omega}(X) & \\
= & 2 \sigma_{\boldsymbol{r}}^{X}\left(s_{1}^{+}\right) \sum_{i=1}^{m}(-1)^{i}\left[\frac{\left\langle\dot{\omega}\left(X-\boldsymbol{r}\left(s_{i}\right)\right), \boldsymbol{u}_{\boldsymbol{r}\left(s_{i}\right)}^{\perp}(X)\right\rangle}{\left|X-\boldsymbol{r}\left(s_{i}\right)\right|}+\frac{\omega\left(X-\boldsymbol{r}\left(s_{i}\right)\right)}{\left|X-\boldsymbol{r}\left(s_{i}\right)\right|^{3}} \bar{\kappa}_{i}^{\boldsymbol{w}}(X)\right]+ \\
& +2 \sigma_{\boldsymbol{r}}^{X}\left(s_{0}^{+}\right) \frac{\left\langle\dot{\omega}\left(X-\boldsymbol{r}\left(s_{0}\right)\right), \boldsymbol{u}_{\boldsymbol{r}\left(s_{0}\right)}^{\perp}(X)\right\rangle}{\left|X-\boldsymbol{r}\left(s_{0}\right)\right|}- \\
& -2 \sigma_{\boldsymbol{r}}^{X}\left(s_{m}^{+}\right) \frac{\left\langle\dot{\omega}\left(X-\boldsymbol{r}\left(s_{m+1}\right)\right), \boldsymbol{u}_{\boldsymbol{r}\left(s_{m+1}\right)}^{\perp}(X)\right\rangle}{\left|X-\boldsymbol{r}\left(s_{m+1}\right)\right|}
\end{aligned}
$$


where

$$
\begin{aligned}
\bar{\kappa}_{i}^{\boldsymbol{w}}(X) & =\frac{\left\langle\boldsymbol{w}^{\perp}, \boldsymbol{u}_{\boldsymbol{r}\left(s_{i}\right)}(X)\right\rangle\left\langle\dot{\boldsymbol{r}}^{\perp}\left(s_{i}\right), \boldsymbol{w}\right\rangle}{\kappa^{e}\left(s_{i}\right)}-\frac{\left\langle\boldsymbol{w}, \boldsymbol{u}_{\boldsymbol{r}\left(s_{i}\right)}(X)\right\rangle\left\langle\dot{\boldsymbol{r}}^{\perp}\left(s_{i}\right), \boldsymbol{w}^{\perp}\right\rangle}{\kappa^{e}\left(s_{i}\right)} \\
& =\frac{-\left\langle\boldsymbol{w}^{\perp}, \boldsymbol{u}_{\boldsymbol{r}\left(s_{i}\right)}(X)\right\rangle\left\langle\dot{\boldsymbol{r}}\left(s_{i}\right), \boldsymbol{w}^{\perp}\right\rangle-\left\langle\boldsymbol{w}, \boldsymbol{u}_{\boldsymbol{r}\left(s_{i}\right)}(X)\right\rangle\left\langle\dot{\boldsymbol{r}}\left(s_{i}\right), \boldsymbol{w}\right\rangle}{\kappa^{e}\left(s_{i}\right)} \\
& =\frac{-\left\langle\dot{\boldsymbol{r}}\left(s_{i}\right), \boldsymbol{u}_{\boldsymbol{r}\left(s_{i}\right)}(X)\right\rangle}{\kappa^{e}\left(s_{i}\right)} .
\end{aligned}
$$

This was stated in the theorem.

Observe, again, that the result of (3.6) does not really depend on $\sigma_{\boldsymbol{r}}^{X}\left(s_{1}^{+}\right)$.

\section{Every $d$-weighted masking function determines its multicurve}

Determination of a multicurve by its $d$-weighted masking function needs several steps. First, we search the traced lines.

Lemma 4.1. Let $\boldsymbol{r}_{\mathcal{J}}$ be a multicurve of class $\mathfrak{C}$.

(1) If no traced line goes through $X$, then $\partial_{\boldsymbol{w}} \bar{M}_{\boldsymbol{r}_{\mathcal{J}} ; \omega}(X)+\partial_{-\boldsymbol{w}} \bar{M}_{\boldsymbol{r}_{\mathcal{J}} ; \omega}(X)=0$.

(2) If $X \notin \operatorname{Tr} \boldsymbol{r}_{\mathcal{J}}$, then $\partial_{\boldsymbol{w}} \bar{M}_{\boldsymbol{r}_{\mathcal{J}} ; \omega}(X)+\partial_{-\boldsymbol{w}} \bar{M}_{\boldsymbol{r}_{\mathcal{J}} ; \omega}(X) \geq 0$, and it is positive if and only if $X$ is on a traced straight line.

(3) On a traced segment of $\boldsymbol{r}_{\mathcal{J}}$ we have $\partial_{\boldsymbol{w}} \bar{M}_{\boldsymbol{r}_{\mathcal{J}} ; \omega}(X)+\partial_{-\boldsymbol{w}} \bar{M}_{\boldsymbol{r}_{\mathcal{J}} ; \omega}(X) \neq 0$ except for finitely many points $X$ of the traced segment.

Proof. Any curve in the class $\mathfrak{C}$ can be partitioned into a finite set of curves so that any such curve is either a segment, or of class $\tilde{\mathfrak{C}}$, therefore we may assume that $\boldsymbol{r}_{\mathcal{J}}$ consists of segments and curves of class $\tilde{\mathfrak{C}}$.

If the curve $\boldsymbol{r}$ is of class $\tilde{\mathfrak{C}}$, then Proposition 3.1 gives $\partial_{\boldsymbol{w}} \bar{M}_{\boldsymbol{r}_{\mathcal{J}} ; \omega}(X)+$ $\partial_{-\boldsymbol{w}} \bar{M}_{\boldsymbol{r}_{\mathcal{J}} ; \omega}(X)=0$ for any point $X$.

If $X \notin \operatorname{Tr} \boldsymbol{r}_{\mathcal{J}}$ is on a traced straight line, then Proposition 3.2 shows $\partial_{\boldsymbol{w}} \bar{M}_{\boldsymbol{r}_{\mathcal{J}} ; \omega}(X)=\partial_{-\boldsymbol{w}} \bar{M}_{\boldsymbol{r}_{\mathcal{J}} ; \omega}(X)>0$.

These prove the first and second claims of the theorem.

If $X$ is in a traced segment $\overline{A B}=\operatorname{Tr} \boldsymbol{r}$ of $\boldsymbol{r} \in \boldsymbol{r}_{\mathcal{J}}$, then Proposition 3.2 shows $\partial_{\boldsymbol{w}} \bar{M}_{\boldsymbol{r} ; \omega}(X)=\partial_{-\boldsymbol{w}} \bar{M}_{\boldsymbol{r} ; \omega}(X)<0$. Thus, to have $\partial_{\boldsymbol{w}} \bar{M}_{\boldsymbol{r}_{\mathcal{J}} ; \omega}(X)+\partial_{-\boldsymbol{w}} \bar{M}_{\boldsymbol{r}_{\mathcal{J}} ; \omega}(X)=0$ at a point $X \in \operatorname{Tr} \boldsymbol{r}, X$ needs to be on some further traced straight lines different from line $A B$, or more traced segments should be on $A B$ and summing up the formulas of (3.1) for these segments results in 0 at $X$. Both options can happen for only finitely many times, that proves the third statement of the theorem. 
To find the traced segments of a multicurve $\boldsymbol{r}_{\mathcal{J}}$ we define the function $S_{\boldsymbol{r}_{\mathcal{J}} ; \omega}: \mathbb{R}^{2} \rightarrow \mathbb{R} \cup\{\infty\}$ by

$$
S_{\boldsymbol{r}_{\mathcal{J}} ; \omega}: X \mapsto \int_{\mathbb{S}^{1}} \partial_{\boldsymbol{w}} \bar{M}_{\boldsymbol{r}_{\mathcal{J}} ; \omega}(X) d \boldsymbol{w} .
$$

Using (3.1) of Proposition 3.2 and observing that $2=\int_{\mathbb{S}^{1}}|\langle\boldsymbol{u}, \boldsymbol{w}\rangle| d \boldsymbol{w}$ for any $\boldsymbol{u} \in \mathbb{S}^{1}$, one easily obtains that

$$
\begin{aligned}
S_{\boldsymbol{r}_{\mathcal{J}} ; \omega}(X)=4 \sum_{i=1}^{k} \omega\left(\boldsymbol{u}_{A_{i}}\left(B_{i}\right)\right)\left|\frac{1}{\left|X-A_{i}\right|}-\frac{1}{\left|X-B_{i}\right|}\right|- \\
\quad-4 \sum_{i=k+1}^{m} \omega\left(\boldsymbol{u}_{A_{i}}\left(B_{i}\right)\right)\left|\frac{1}{\left|X-A_{i}\right|}+\frac{1}{\left|X-B_{i}\right|}\right|,
\end{aligned}
$$

where $\overline{A_{i} B_{i}}$ are the traced segments of $\boldsymbol{r}_{\mathcal{J}}$ which are collinear with $X, X \notin \overline{A_{i} B_{i}}$ if $i=1, \ldots, k$, and $X \in \overline{A_{i} B_{i}}$ if $i=k+1, \ldots, m$.

This function allows us to detect the traced straight lines.

Proposition 4.2. Let the multicurve $\boldsymbol{r}_{\mathcal{J}}$ be of class $\mathfrak{C}$ and $X \in \mathbb{R}^{2}$. Then for any $\boldsymbol{v} \in \mathbb{S}^{1}$ the function $\hat{S}_{\boldsymbol{r}_{\mathcal{J}} ; \omega}(X, \boldsymbol{v}):=\lim _{0<t \backslash 0} S_{\boldsymbol{r}_{\mathcal{J}} ; \omega}(X+t \boldsymbol{v})$ vanishes if and only if $\ell(X, \boldsymbol{v}) \notin \mathcal{L}_{\boldsymbol{r}_{\mathcal{J}}}$.

Proof. Lemma 4.1 states that $S_{\boldsymbol{r}_{\mathcal{J}} ; \omega}(X+t \boldsymbol{v})$ vanishes if $X+t \boldsymbol{v}$ is not on a traced straight line, therefore $\ell(X, \boldsymbol{v}) \notin \mathcal{L}_{\boldsymbol{r}_{\mathcal{J}}}$ implies that $S_{\boldsymbol{r}_{\mathcal{J}} ; \omega}(X+t \boldsymbol{v}) \neq 0$ for only finitely many values of $t$, hence $\hat{S}_{\boldsymbol{r}_{\mathcal{J}} ; \omega}(X, \boldsymbol{v})=0$.

Suppose that $\ell(X, \boldsymbol{v}) \in \mathcal{L}_{\boldsymbol{r}_{\mathcal{J}}}$.

Except for finitely many values of $t$, the only straight line passing through the point $X+t \boldsymbol{v}$ is $\ell(X, \boldsymbol{v})$, therefore equation (4.1) implies

$$
\begin{aligned}
& \frac{S_{\boldsymbol{r}_{\mathcal{J}} ; \omega}(X+t \boldsymbol{v})}{4 \omega(\boldsymbol{v})} \\
& =\sum_{i=1}^{k}\left|\frac{1}{\left|X+t \boldsymbol{v}-A_{i}\right|}-\frac{1}{\left|X+t \boldsymbol{v}-B_{i}\right|}\right|-\sum_{i=k+1}^{m}\left|\frac{1}{\left|X+t \boldsymbol{v}-A_{i}\right|}+\frac{1}{\left|X+t \boldsymbol{v}-B_{i}\right|}\right|,
\end{aligned}
$$

for $t>0$ small enough. As the right-hand side of this equation ${ }^{10}$ can only vanish in finitely many values of $t, \hat{S}_{\boldsymbol{r}_{\mathcal{J}} ; \omega}(X, \boldsymbol{v}) \neq 0$ follows. The proof is completed.

Having determined the traced segments, we are looking for the points of the multicurve that are not in traced segments. By (2.4) we have

$$
\Delta M_{\boldsymbol{r}_{\mathcal{J}} ; \omega}(X):=\sum_{j \in \mathcal{J}} \Delta M_{\boldsymbol{r}_{j} ; \omega}(X) .
$$

\footnotetext{
${ }^{10}$ Observe that the second sum may be empty or it has only one member.
} 
Proposition 4.3. Let the multicurve $\boldsymbol{r}_{\mathcal{J}}$ be of class $\tilde{\mathfrak{C}}$. Then the function

$$
C_{\boldsymbol{r}_{\mathcal{J}} ; \omega}(X):=\sup _{\boldsymbol{v} \in \mathbb{S}^{1}} \lim _{0<t \searrow 0}\left(t^{3}\left|\Delta M_{\boldsymbol{r}_{\mathcal{J}} ; \omega}(X+t \boldsymbol{v})\right|\right)
$$

vanishes if and only if $X \notin \operatorname{Tr} \boldsymbol{r}_{\mathcal{J}}$ or $X$ is in a finite set of $\operatorname{Tr} \boldsymbol{r}_{\mathcal{J}}$.

Proof. By equation (3.6) it is obvious that $\lim _{t \searrow 0}\left(t^{3}\left|\Delta M_{\boldsymbol{r}_{\mathcal{J}} ; \omega}(X+t \boldsymbol{v})\right|\right)$ vanishes if $X \notin \operatorname{Tr} \boldsymbol{r}_{\mathcal{J}}$.

To prove the statement, we need to show for every curve $\boldsymbol{r} \in \boldsymbol{r}_{\mathcal{J}}$ that, except for a finite set of points $X$ in $\operatorname{Tr} \boldsymbol{r}$, there is a vector $\boldsymbol{v} \in \mathbb{S}^{1}$ so that $\lim _{0<t \backslash 0}\left(t^{3}\left|\Delta M_{\boldsymbol{r} ; \omega}(X+t \boldsymbol{v})\right|\right)$ does not vanish. Thus, it is enough to consider those points of $\operatorname{Tr} \boldsymbol{r}$, where the curvature is not zero.

Assume now that $X=\boldsymbol{r}(s)$ and $\ddot{\boldsymbol{r}}(s) \neq \mathbf{0}$. Let $\boldsymbol{v}=\dot{\boldsymbol{r}}(s)$ and $Y=X+t \boldsymbol{v}$ for small values of $t>0$. Taking equation (3.6) into account, one can see that only those members of the sum in (3.6) may play a role in $\lim _{0<t \backslash 0}\left(t^{3}\left|\Delta M_{r ; \omega}(Y)\right|\right)$, where $\boldsymbol{r}\left(s_{i}(Y)\right) \rightarrow X=\boldsymbol{r}(s)$ ( $i$ is an index in the sum) as $t \rightarrow 0$. As $\boldsymbol{r}$ is not self-intersecting, this implies $s_{i}(Y) \rightarrow s$ along $t \rightarrow 0$. As $\ddot{\boldsymbol{r}}(s) \neq \mathbf{0}$, only two consecutive indices may satisfy this. So to say $s=s_{i-1}(Y)$ and $s_{i}(Y) \rightarrow s$. Then by Theorem 3.5, we have

$$
\begin{aligned}
& \lim _{0<t \searrow 0} \frac{t^{3}\left|\Delta M_{\boldsymbol{r} ; \omega}(Y)\right|}{2(-1)^{i} \sigma_{\boldsymbol{r}}^{Y}\left(s_{1}^{+}(Y)\right)} \\
& \quad=\lim _{0<t \searrow 0}\left(\frac{t^{3} \omega\left(Y-\boldsymbol{r}\left(s_{i-1}(Y)\right)\right)}{\left|Y-\boldsymbol{r}\left(s_{i-1}(Y)\right)\right|^{3} \kappa^{e}\left(s_{i-1}(Y)\right)}+\frac{t^{3} \omega\left(Y-\boldsymbol{r}\left(s_{i}(Y)\right)\right)}{\left|Y-\boldsymbol{r}\left(s_{i}(Y)\right)\right|^{3} \kappa^{e}\left(s_{i}(Y)\right)}\right) \\
& \quad=\frac{\omega(\boldsymbol{v})}{\kappa^{e}(s)} .
\end{aligned}
$$

This proves the theorem.

Now we are ready to prove the following Theorem 4.4 which is analogous to [2, Theorem 10.1], [17, Theorem 1.1] and [16, Theorem 2.1]. Compared to these results our one is less general in the sense that it requests the multicurves to be in $\mathfrak{C}$, but it is more general in the sense that it allows any d-weight not just the constant 1.

Theorem 4.4. Any d-weighted masking function of a multicurve of class $\mathfrak{C}$ determines the trace of the multicurve.

Proof. Any curve in $\mathfrak{C}$ can be partitioned into a finite set of curves so that any one of those curves is either a segment, or is in $\tilde{\mathfrak{C}}$, therefore we may assume that every 
member of the unknown multicurve $\boldsymbol{r}_{\mathcal{J}}$ of the known d-weighted masking function $M_{\boldsymbol{r}_{\mathcal{J}} ; \omega}$ is either a segment or is in $\tilde{\mathfrak{C}}$.

First we determine the segments.

Proposition 4.2 implies that

$$
\bigcup_{\ell \in \mathcal{L}_{\boldsymbol{r}_{\mathcal{J}}}} \ell=\left\{X \in \mathbb{R}^{2}: 0<\sup _{\boldsymbol{v} \in \mathbb{S}^{1}}\left|\hat{S}_{\boldsymbol{r}_{\mathcal{J}} ; \boldsymbol{\omega}}(X, \boldsymbol{v})\right|\right\} .
$$

This allows us to choose a vector $\boldsymbol{v} \in \mathbb{S}^{1}$ for each point $X \in \bigcup_{\ell \in \mathcal{L}_{r_{\mathcal{J}}}} \ell$ such that $\left|\hat{S}_{\boldsymbol{r}_{\mathcal{J}} ; \omega}(X, \boldsymbol{v})\right|$ is positive. Then $\hat{S}_{\boldsymbol{r}_{\mathcal{J}} ; \omega}(X, \boldsymbol{v})$ is either infinite, in which case $X \in$ $\operatorname{Tr} \boldsymbol{r}_{\mathcal{J}}$ is an endpoint of a maximal traced segment, or $\hat{S}_{\boldsymbol{r}_{\mathcal{J}} ; \omega}(X, \boldsymbol{v})$ is finite. If this is the case, then, by (4.2), we have for a small $\varepsilon>0$ and every $t \in(-\varepsilon, \varepsilon)$ that

$$
\frac{\hat{S}_{\boldsymbol{r}_{\mathcal{J}} ; \omega}(X+t \boldsymbol{v}, \boldsymbol{v})}{4 \omega(\boldsymbol{v})}=\left\{\begin{array}{cl}
\sum_{i=1}^{k}\left|\frac{1}{\left|X+t \boldsymbol{v}-A_{i}\right|}-\frac{1}{\left|X+t \boldsymbol{v}-B_{i}\right|}\right|, & \text { if } X \notin \operatorname{Tr} \boldsymbol{r}_{\mathcal{J}}, \\
\sum_{i=1}^{k}\left|\frac{1}{\left|X+t \boldsymbol{v}-A_{i}\right|}-\frac{1}{\left|X+t \boldsymbol{v}-B_{i}\right|}\right|+ & \text { if, say, } \\
+\left(\frac{1}{\left|X+t \boldsymbol{v}-A_{k+1}\right|}+\frac{1}{\left|X+t \boldsymbol{v}-B_{k+1}\right|}\right), & X \in \overline{A_{k+1} B_{k+1}},
\end{array}\right.
$$

where $\overline{A_{i} B_{i}}(i=1, \ldots, m)$ are the maximal traced segments on $\ell(X, \boldsymbol{v})$. In both cases of (4.4) we can apply Theorem A.1 to find $\omega(\boldsymbol{v})$ and every $X-A_{i}$ and $X-B_{i}$, hence obtaining segments of $\boldsymbol{r}_{\mathcal{J}}$ on $\ell(X, \boldsymbol{v})$.

Thus, choosing $X \in \bigcup_{\ell \in \mathcal{L}_{\boldsymbol{r}_{\mathcal{J}}}} \ell$ and $\boldsymbol{v} \in \mathbb{S}^{1}$ so that $\left|\hat{S}_{\boldsymbol{r}_{\mathcal{J}} ; \omega}(X, \boldsymbol{v})\right|$ is positive and the segments of $\boldsymbol{r}_{\mathcal{J}}$ on $\ell(X, \boldsymbol{v})$ is not known, determines every traced segment within finite steps.

Let $\boldsymbol{r}_{\mathcal{S}}$ be the multicurve made up from the segments of $\boldsymbol{r}_{\mathcal{J}}$, and let $\boldsymbol{r}_{\mathcal{K}}$ be the other curves in $\boldsymbol{r}_{\mathcal{J}}$. Then we clearly have $\bar{M}_{\boldsymbol{r}_{\mathcal{K}} ; \omega}=\bar{M}_{\boldsymbol{r}_{\mathcal{J}} ; \omega}-\bar{M}_{\boldsymbol{r}_{\mathcal{S}} ; \omega}$. As $\boldsymbol{r}_{\mathcal{K}}$ is of class $\tilde{\mathfrak{C}}$, Proposition 4.3 says that $\operatorname{supp} C_{\boldsymbol{r}_{\mathcal{K}} ; \omega}$ is a subset of $\operatorname{Tr} \boldsymbol{r}_{\mathcal{K}}$ so that $\operatorname{Tr} \boldsymbol{r}_{\mathcal{K}} \backslash \operatorname{supp} C_{\boldsymbol{r}_{\mathcal{K}} ; \omega}$ is a finite set (maybe empty).

Closing topologically the set $\operatorname{supp} C_{\boldsymbol{r}_{\mathcal{K}} ; \omega}$ gives $\operatorname{Tr} \boldsymbol{r}_{\mathcal{K}}$ and completes the proof.

\section{Recover multicurves from their restricted masking functions}

Having Theorem 4.4, the question arises if it may be enough to know the masking functions on a smaller set and still be able to determine the multicurves. This is the goal of this section.

We consider the masking functions restricted to a ring, therefore in this section $\mathcal{D} \subset \mathbb{R}^{2}$ is a compact domain and $\mathcal{R}$ is an open (not necessarily circular) ring around $\mathcal{D}$. 
Theorem 5.1. If the d-weighted masking function of a multicurve $\boldsymbol{r}_{\mathcal{J}} \in \mathfrak{C}^{\mathcal{D}}$ is known in $\mathcal{R}$, then the traced segments of $\boldsymbol{r}_{\mathcal{J}}$ can be determined

Proof. First we observe (4.3) in the proof of Theorem 4.4 and see that we are able to determine $\mathcal{R} \cap \bigcup_{\ell \in \mathcal{L}_{r_{\mathcal{J}}}} \ell$.

As every traced straight line $\ell \in \mathcal{L}_{\boldsymbol{r}_{\mathcal{J}}}$ intersects $\mathcal{R}$ in a segment, (4.4) and the method that follows it in the proof of Theorem 4.4 show that we can determine every traced segment of $\boldsymbol{r}_{\mathcal{J}}$.

By this result it can be decided if a multicurve $\boldsymbol{r}_{\mathcal{J}}$ is a multisegment by simply considering its masking function. For, we determine its traced segments and subtract the masking function of the multisegment composed of these segments from the masking function of $\boldsymbol{r}_{\mathcal{J}}$. If this difference is identically zero, then $\boldsymbol{r}_{\mathcal{J}}$ is a multisegment, otherwise it has more curves of class $\tilde{\mathfrak{C}}^{\mathcal{D}}$.

Having the masking function of a multicurve $\boldsymbol{r}_{\mathcal{J}}$ for a complete independent set of d-weights makes the trace of the multicurve recoverable, because in certain circumstances it determines the function $\alpha \mapsto \#\left(\operatorname{Tr} \boldsymbol{r}_{\mathcal{J}} \cap \ell\left(X, \boldsymbol{u}_{\alpha}\right)\right)$ at every given point $X$ and then Theorem 4.4 gives $\operatorname{Tr} \boldsymbol{r}_{\mathcal{J}}$. The following result offers a direct way of determination.

Theorem 5.2. If there is a unit vector $\boldsymbol{v}_{\omega} \in \mathbb{S}^{1}$ so that the d-weights $\omega_{n}(n \in \mathbb{N})$ can be written in the form $\omega_{n}(X)=\left|\left\langle X /|X|, \boldsymbol{v}_{\omega}\right\rangle\right|^{n}$, then the trace of any multicurve $\boldsymbol{r}_{\mathcal{J}} \in \mathfrak{C}^{\mathcal{D}}$ can be determined by having $\bar{M}_{\boldsymbol{r}_{\mathcal{J}} ; \omega_{n}}$ on $\mathcal{R}$ for every $n \in \mathbb{N}$.

Proof. Any one of the d-weighted masking functions $\bar{M}_{\boldsymbol{r}_{\mathcal{J}} ; \omega_{n}}$ determines the segments in $\operatorname{Tr} \boldsymbol{r}_{\mathcal{J}}$ by Theorem 5.1. Removing the maximal traced segments from $\boldsymbol{r}_{\mathcal{J}}$, it remains to prove the statement for the resulting multicurve that is of class $\tilde{\mathfrak{C}}$.

Therefore, from now on we assume that $\boldsymbol{r}_{\mathcal{J}} \in \tilde{\mathfrak{C}}^{\mathcal{D}}$. Further, by properly partitioning $\boldsymbol{r}_{\mathcal{J}}$ we assume also that each member curve of $\boldsymbol{r}_{\mathcal{J}}$ has non-vanishing curvature at internal points.

Now take a member curve $r:[a, b] \rightarrow \mathcal{D}$, choose a point $X \in \mathcal{R}$ and let $\hat{\Theta}_{r}^{X}=\left\{s_{i}\right\}_{i=1}^{m} \subset \mathbb{R}_{\boldsymbol{r}}=\left[s_{0}, s_{m+1}\right]$, where the values $s_{i}(i=0, \ldots, m+1)$ are indexed so that Theorem 3.3 gives

$$
\begin{aligned}
\hat{\boldsymbol{M}}_{\boldsymbol{r} ; \omega_{n}}(X): & =\partial_{\boldsymbol{w}} \bar{M}_{\boldsymbol{r} ; \omega_{n}}(X) \boldsymbol{w}^{\perp}-\partial_{\boldsymbol{w}^{\perp}} \bar{M}_{\boldsymbol{r} ; \omega_{n}}(X) \boldsymbol{w} \\
& =\sum_{i=0}^{m+1} \omega_{n}\left(X-\boldsymbol{r}\left(s_{i}\right)\right) \boldsymbol{N}_{i}(X) \boldsymbol{u}_{\boldsymbol{r}\left(s_{i}\right)}(X),
\end{aligned}
$$

where

$$
\boldsymbol{N}_{i}(X)=\frac{1}{\left|X-\boldsymbol{r}\left(s_{i}\right)\right|} \times \begin{cases}-\sigma_{\boldsymbol{r}}^{X}\left(s_{0}^{+}\right), & \text {if } i=0, \\ 2(-1)^{i} \sigma_{\boldsymbol{r}}^{X}\left(s_{1}^{+}\right), & \text {if } 1 \leq i \leq m, \\ \sigma_{\boldsymbol{r}}^{X}\left(s_{m}^{+}\right), & \text {if } i=m+1\end{cases}
$$


Take the polynomials

$$
b_{k}(t):=\frac{2 k+1}{2} B_{k}^{2 k}\left(\frac{t+1}{2}\right)=\frac{2 k+1}{2^{2 k+1}}\left(\begin{array}{c}
2 k \\
k
\end{array}\right)\left(1-t^{2}\right)^{k},
$$

where $B_{k}^{2 k}$ is the $k$ th Bernstein base polynomial of degree $k \in \mathbb{N}$. Then there clearly exists a unique vector $\boldsymbol{b}_{x, k} \in \mathbb{R}^{2 k+1}$ for which $b_{k}(t-x)=\left\langle\boldsymbol{b}_{x, k},\left(1, t, t^{2}, \ldots, t^{2 k}\right)\right\rangle$, hence we have

$$
\begin{aligned}
\boldsymbol{B}_{k}(X, x): & =\left\langle\boldsymbol{b}_{x, k},\left(\hat{\boldsymbol{M}}_{\boldsymbol{r} ; \omega_{0}}(X), \hat{\boldsymbol{M}}_{\boldsymbol{r} ; \omega_{1}}(X), \ldots, \hat{\boldsymbol{M}}_{\boldsymbol{r} ; \omega_{2 k}}(X)\right)\right\rangle \\
& =\sum_{i=0}^{m+1} b_{k}\left(\omega_{1}\left(\boldsymbol{u}_{\boldsymbol{r}\left(s_{i}\right)}(X)\right)-x\right) \boldsymbol{N}_{i}(X) \boldsymbol{u}_{\boldsymbol{r}\left(s_{i}\right)}(X)=\hat{\boldsymbol{M}}_{\boldsymbol{r} ; b_{k}\left(\omega_{1}(\cdot)-x\right)}(X) .
\end{aligned}
$$

As the sequence $k \mapsto b_{k}$ is delta-convergent ${ }^{11}$ on $(-1,1)$, we obtain

$$
\boldsymbol{f}_{X}(x):=\lim _{k \rightarrow \infty} \boldsymbol{B}_{k}(X, x)=\sum_{i \in \mathcal{I}_{X}(x)} \boldsymbol{N}_{i}(X) \boldsymbol{u}_{\boldsymbol{r}\left(s_{i}\right)}(X),
$$

where $\mathcal{I}_{X}(x):=\left\{i: x=\left|\left\langle\boldsymbol{u}_{\boldsymbol{r}\left(s_{i}\right)}(X), \boldsymbol{v}_{\omega}\right\rangle\right|\right\}$ for any $x \in(0,1)$, and an empty sum is meant to be $\mathbf{0}$.

Fix $X$ and consider the sets

$$
\mathcal{T}_{i, j}:=\left\{Y:\left\langle\boldsymbol{u}_{\boldsymbol{r}\left(s_{i}(Y)\right)}(Y), \boldsymbol{v}_{\omega}\right\rangle=\left\langle\boldsymbol{u}_{\boldsymbol{r}\left(s_{j}(Y)\right)}(Y), \boldsymbol{v}_{\omega}\right\rangle,|Y-X|<\varepsilon\right\}
$$

for a small $\varepsilon>0$ and different indexes $i, j \in\{0, \ldots, m+1\}$. If $\mathcal{T}_{i, j} \neq \emptyset$, then $\mathcal{T}_{i, j}$ is the trace of a differentiable curve by the implicit function theorem [9]. As only finitely many such curves may exist, there is a neighbourhood $\mathcal{U}$ in any open set that has no common point with $\bigcup_{i \neq j} \mathcal{T}_{i, j}$. Therefore at every point $X \in \mathcal{U}$ the set $\mathcal{I}_{X}(x)$ has at most one element for any $x \in(0,1)$. As the vector $\boldsymbol{u}_{\boldsymbol{r}\left(s_{i}(X)\right)}(X)$ changes continuously with $X$, there is a neighbourhood $\mathcal{U}^{\prime} \subset \mathcal{U}$ so that

$$
\mathcal{A}_{\mathcal{U}^{\prime}}:=\bigcup_{i=0}^{m+1}\left\{\boldsymbol{u}_{\boldsymbol{r}\left(s_{i}(X)\right)}(X): X \in \mathcal{U}^{\prime}\right\}
$$

is a union of disjoint connected $\operatorname{arcs} \mathcal{A}_{j}$ in $\mathbb{S}^{1}$, and each arc $\mathcal{A}_{i}$ contains exactly one unit vector of the form $\boldsymbol{u}_{\boldsymbol{r}\left(s_{i}(X)\right)}(X)$ for every $X \in \mathcal{U}^{\prime}$, hence $i=0, \ldots, m+1$.

Observe that for any $x \in(0,1)$ the set $\mathcal{I}_{X}(x)$ has at most one element at every $X \in \mathcal{U}^{\prime}$, hence

$$
\mathcal{A}_{\mathcal{U}^{\prime}}=\bigcup_{X \in \mathcal{U}^{\prime}}\left\{\frac{\boldsymbol{f}_{X}(x)}{\left|\boldsymbol{f}_{X}(x)\right|}: x \in(0,1), \boldsymbol{f}_{X}(x) \neq \mathbf{0}\right\},
$$

and therefore the $\operatorname{arcs} \mathcal{A}_{i}$ are also determined by the given masking functions.

\footnotetext{
${ }^{11} b_{k}$ tends to the Dirac delta in the dual space of continuous functions [5, Exercise 16 on p. 16].
} 
Fix an $\operatorname{arc} \mathcal{A}_{i}$ and choose at every $X \in \mathcal{U}^{\prime}$ the unique $x_{i} \in(0,1)$ for which $\boldsymbol{f}_{X}\left(x_{i}\right) \neq \mathbf{0}$ and $\frac{\boldsymbol{f}_{X}\left(x_{i}\right)}{\left|\boldsymbol{f}_{X}\left(x_{i}\right)\right|} \in \mathcal{A}_{i}$. Then for the appropriate $i \in\{0, \ldots, m+1\}$ we have $x_{i}=\left|\left\langle\boldsymbol{u}_{\boldsymbol{r}\left(s_{i}\right)}(X), \boldsymbol{v}\right\rangle\right|$ and the unit vector $\boldsymbol{u}_{\boldsymbol{r}\left(s_{i}(X)\right)}(X)=\frac{\boldsymbol{f}_{X}\left(x_{i}\right)}{\left|\boldsymbol{f}_{X}\left(x_{i}\right)\right|}$ as a function of $X \in \mathcal{U}^{\prime}$. In addition to these we also have $\left|X-\boldsymbol{r}\left(s_{i}(X)\right)\right|=1 /\left|\boldsymbol{f}_{X}\left(x_{i}\right)\right|$, hence

$$
\boldsymbol{r}\left(s_{i}(X)\right)=X+\frac{1}{\left|\boldsymbol{f}_{X}\left(x_{i}\right)\right|} \boldsymbol{u}_{\boldsymbol{r}\left(s_{i}(X)\right)}(X) .
$$

As $X$ freely runs over the ring $\mathcal{R}$, except for the finitely many curves given by (5.1) and the tangents of $\boldsymbol{r}$ at the endpoints, (5.2) determines the trace of $\boldsymbol{r}$ as the closure of the points of the trace, where the curvature does not vanish.

For the multicurve $\boldsymbol{r}_{\mathcal{J}} \in \tilde{\mathfrak{C}}$, to determine its trace, the procedure remains the same as above by just changing $\boldsymbol{r}$ to $\boldsymbol{r}_{\mathcal{J}}$ in the formulas, because every time the reasoning was based on the finiteness of a set, the corresponding set for the multicurve is also finite. The theorem is proved.

Notice that the proof gives $\left\langle\boldsymbol{u}_{\boldsymbol{r}\left(s_{i}\right)}(X), \boldsymbol{v}_{\omega}\right\rangle$ for every $\boldsymbol{u}_{\boldsymbol{r}\left(s_{i}\right)}(X)$.

\section{Proof of the conjecture for analytic multicurves}

In this section we call $\bar{M}_{\boldsymbol{r}_{\mathcal{J}}}:=\bar{M}_{\boldsymbol{r}_{\mathcal{J}} ; 1}$ simply the (weightless) masking function. Observe that $\omega \equiv 1$ simplifies equation (3.6) into

$$
\begin{aligned}
\Delta M_{\boldsymbol{r}}(X) & =\sigma_{\boldsymbol{r}}^{X}\left(s_{1}^{+}\right) \sum_{i=1}^{m}(-1)^{i-1} \frac{\left\langle\boldsymbol{u}_{\boldsymbol{r}\left(s_{i}\right)}(X), \dot{\boldsymbol{r}}\left(s_{i}\right)\right\rangle}{\left|X-\boldsymbol{r}\left(s_{i}\right)\right|^{3} \kappa^{e}\left(s_{i}\right)} \\
& =\sigma_{\boldsymbol{r}}^{X}\left(s_{1}^{+}\right) \sum_{i=1}^{m} \frac{(-1)^{i-1}}{\left|X-\boldsymbol{r}\left(s_{i}\right)\right|^{3}} \frac{\left\langle\boldsymbol{u}_{\boldsymbol{r}\left(s_{i}\right)}^{\perp}(X), \dot{\boldsymbol{r}}^{\perp}\left(s_{i}\right)\right\rangle}{\kappa\left(s_{i}\right) \operatorname{sign}\left(\left\langle\ddot{\boldsymbol{r}}\left(s_{i}\right), \dot{\boldsymbol{r}}^{\perp}\left(s_{i}\right)\right\rangle\right)} \\
& =\sigma_{\boldsymbol{r}}^{X}\left(s_{1}^{+}\right) \sum_{i=1}^{m} \frac{(-1)^{i-1} \operatorname{sign}\left(\left\langle\boldsymbol{u}_{\boldsymbol{r}\left(s_{i}\right)}^{\perp}(X), \ddot{\boldsymbol{r}}\left(s_{i}\right)\right\rangle\right)}{\left|X-\boldsymbol{r}\left(s_{i}\right)\right|^{3} \kappa\left(s_{i}\right)} .
\end{aligned}
$$
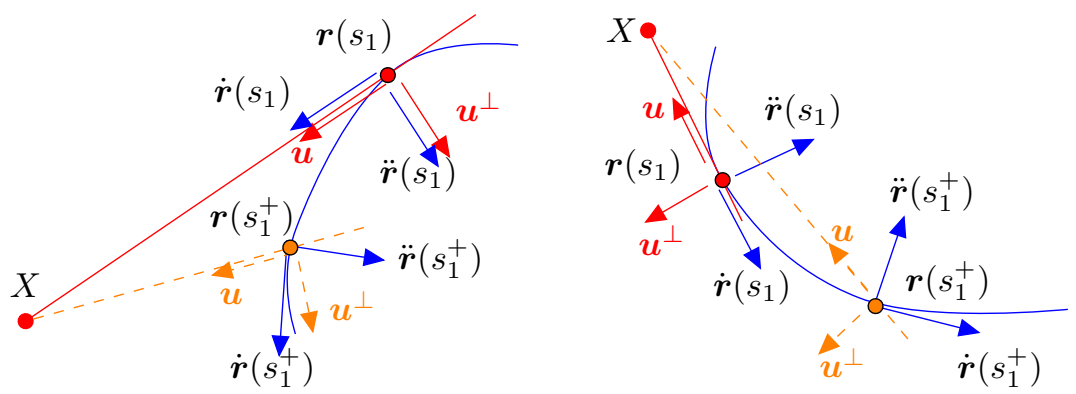

Figure 6.1. Two of the four possible placings of $X, \boldsymbol{r}$ and $\dot{\boldsymbol{r}}$ are pictured here to demonstrate (6.1) and (6.2) 
Taking into account (see Figure 6.1 for a demonstration) that

$$
(-1)^{i-1} \operatorname{sign}\left(\left\langle\boldsymbol{u}_{\boldsymbol{r}\left(s_{i}\right)}^{\perp}(X), \ddot{\boldsymbol{r}}\left(s_{i}\right)\right\rangle\right)=\operatorname{sign}\left(\left\langle\boldsymbol{u}_{\boldsymbol{r}\left(s_{1}\right)}^{\perp}(X), \ddot{\boldsymbol{r}}\left(s_{1}\right)\right\rangle\right)
$$

and

$$
\sigma_{\boldsymbol{r}}^{X}\left(s_{1}^{+}\right)=\operatorname{sign}\left(\left\langle\dot{\boldsymbol{r}}\left(s_{1}^{+}\right), \boldsymbol{u}_{\boldsymbol{r}\left(s_{1}^{+}\right)}^{\perp}(X)\right\rangle\right)=\operatorname{sign}\left(\left\langle\boldsymbol{u}_{\boldsymbol{r}\left(s_{1}\right)}^{\perp}(X), \ddot{\boldsymbol{r}}\left(s_{1}\right)\right\rangle\right),
$$

we arrive at the equality ${ }^{12}$

$$
\Delta M_{\boldsymbol{r}}(X)=\sum_{i=1}^{m} \frac{1 / \kappa\left(s_{i}(X)\right)}{\left|X-\boldsymbol{r}\left(s_{i}(X)\right)\right|^{3}}
$$

that proves the following.

Theorem 6.1. Except at the points where it is not differentiable,

(1) the masking function of every multisegment is locally harmonic,

(2) the masking function of every multicurve is locally subharmonic.

Let $\mathfrak{E}$ be the set of those multicurves $\boldsymbol{r}_{\mathcal{J}}$ in $\mathfrak{C}$ whose every member curve $\boldsymbol{r}_{j}$ is a restriction of an entire function $\tilde{\boldsymbol{r}}_{j}$ that is either linear or has positive curvature everywhere and traces the border of a convex (not necessarily compact) domain $\mathcal{K}_{j}$. We say that the curves $\boldsymbol{r}_{j}, \boldsymbol{r}_{i} \in \mathfrak{E}$ are tangent of order $0<k \in \mathbb{N}$ at a common point $\boldsymbol{r}_{j}(\bar{s})=\boldsymbol{r}_{i}(s)$, if for a suitable sign $\boldsymbol{r}_{j}^{\prime}(\bar{s})= \pm \boldsymbol{r}_{i}^{\prime}(s), \ldots, \boldsymbol{r}_{j}^{(k)}(\bar{s})= \pm \boldsymbol{r}_{i}^{(k)}(s) .{ }^{13}$

Theorem 6.2. Let $\mathcal{D} \subset \mathbb{R}^{2}$ be a compact domain and let $\mathcal{R}$ be an open ring around $\mathcal{D}$. Let the multicurve $\boldsymbol{r}_{\mathcal{J}} \in \mathfrak{E}$ be $i n^{14} \mathcal{D}$ such that no two of its member curves are tangent of order two at any of their endpoints. If $\bar{M}_{\boldsymbol{r}_{\mathcal{J}}}$ is known on $\mathcal{R}$, then the trace of $\boldsymbol{r}_{\mathcal{J}}$ can be recovered. ${ }^{15}$

Proof. By Theorem 5.1, the restriction of the masking function $\bar{M}_{\boldsymbol{r}_{\mathcal{J}} ; \omega}$ to $\mathcal{R}$ determines the segments in $\operatorname{Tr} \boldsymbol{r}_{\mathcal{J}}$, so by removing the maximal traced segments we may assume from now on that the unique analytic extensions of the member curves of $\boldsymbol{r}_{\mathcal{J}}$ have positive curvature everywhere.

Let $\boldsymbol{r}:[a, b] \rightarrow \mathcal{D}$ be a member curve of $\boldsymbol{r}_{\mathcal{J}}$, and let $\tilde{\boldsymbol{r}}$ denote its unique analytic extension. Denote by $\mathcal{K}$ the convex domain the border of which is traced by $\tilde{\boldsymbol{r}}$, i.e. $\partial \mathcal{K}=\operatorname{Tr} \tilde{\boldsymbol{r}}$. Let $\ell_{a}:=\ell\left(\boldsymbol{r}(a), \dot{\boldsymbol{r}}\left(a^{+}\right)\right)$and $\ell_{b}:=\ell\left(\boldsymbol{r}(b), \dot{\boldsymbol{r}}\left(b^{-}\right)\right)$. If $\ell_{a} \neq \ell_{b}$, then let $\mathcal{E}_{r}:=\ell_{a} \cup \ell_{b}$, otherwise set $\mathcal{E}_{\boldsymbol{r}}=\emptyset$.

\footnotetext{
${ }^{12}$ For convex curves this can be easily proved by the second formula of [10, Lemma 1].

${ }^{13}$ If $\boldsymbol{r}_{j}(\bar{s})$ or $\boldsymbol{r}_{i}(s)$ is an endpoint of $\boldsymbol{r}_{j}$ resp. $\boldsymbol{r}_{i}$, then these derivatives are understood as left- or right-derivatives.

${ }^{14}$ This does not imply $\mathcal{K}_{j} \subseteq \mathcal{D}$.

${ }^{15}$ In fact we prove that every member curve $\boldsymbol{r}_{j}$ can be recovered up to an unknown shift in its argument.
} 
Assume that $X_{0} \notin \mathcal{E}_{\boldsymbol{r}} \cup \operatorname{Tr} \boldsymbol{r}$ and $\hat{\Theta}_{r}^{X_{0}}$ is not empty. Then we have $\hat{\Theta}_{r}^{X_{0}}=\left\{s_{i}\left(X_{0}\right)\right\}_{i=1}^{m} \subset(a, b)$ for an $m \in\{1,2\}$, where $s_{1}\left(X_{0}\right)<s_{2}\left(X_{0}\right)$. In a neighbourhood of $X_{0}$ every function $s_{i}: X \mapsto s_{i}(X)$ is a solution of the equation $\left\langle X-\boldsymbol{r}(s), \dot{\boldsymbol{r}}^{\perp}\left(s_{i}(X)\right)\right\rangle=0$ the derivative of which at a solution $\xi=\left(X_{0}, s_{i}\left(X_{0}\right)\right)$ is

$$
\begin{aligned}
\left.\frac{\partial\left\langle X-\boldsymbol{r}(s), \dot{\boldsymbol{r}}^{\perp}(s)\right\rangle}{\partial s}\right|_{\xi} & =\left\langle X_{0}-\boldsymbol{r}\left(s_{i}\right), \ddot{\boldsymbol{r}}^{\perp}\left(s_{i}\right)\right\rangle=-\kappa^{e}\left(s_{i}\right)\left\langle X_{0}-\boldsymbol{r}\left(s_{i}\right), \dot{\boldsymbol{r}}\left(s_{i}\right)\right\rangle \\
& = \pm \kappa^{e}\left(s_{i}\right)\left|X_{0}-\boldsymbol{r}\left(s_{i}\right)\right| \neq 0, \\
\left|\frac{\partial\left\langle X-\boldsymbol{r}(s), \dot{\boldsymbol{r}}^{\perp}(s)\right\rangle}{\partial X}\right|_{\xi} & =\left|\dot{\boldsymbol{r}}^{\perp}\left(s_{i}\right)\right|=1 \neq 0 .
\end{aligned}
$$

Thus, by the analytic implicit function theorem [9], the functions $s_{i}$ are real analytic in a neighbourhood of any such $X_{0} \notin \mathcal{E}_{\boldsymbol{r}} \cup \operatorname{Tr} \boldsymbol{r}$.

Taking (6.3) into account we infer that $\Delta M_{r}$ is real analytic on every connected domain of $\mathbb{R}^{2} \backslash\left(\mathcal{E}_{\boldsymbol{r}} \cup \operatorname{Tr} \boldsymbol{r}\right)$.

As $\tilde{\boldsymbol{r}}$ has curvature everywhere positive, we infer that either $\ell_{a} \neq \ell_{b}$ and $\boldsymbol{r}(a) \neq \boldsymbol{r}(b)$ or $\ell_{a}=\ell_{b}$ and $\boldsymbol{r}(a)=\boldsymbol{r}(b)$.

First, we suppose $\boldsymbol{r}(a) \neq \boldsymbol{r}(b)$ and $\ell_{a} \neq \ell_{b}$.

In this case $\Delta M_{r}$ is not continuous at the points of $\mathcal{E}_{r}$. To see this, let $X_{0} \in \mathcal{E}_{\boldsymbol{r}} \backslash \operatorname{Tr} \boldsymbol{r}$ differ from $I:=\ell_{a} \cap \ell_{b}$.
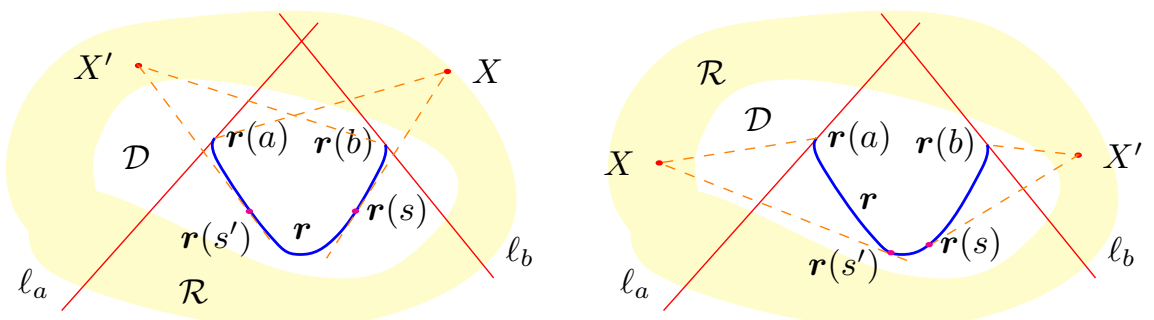

Figure 6.2. Discontinuity of $\Delta M_{r}$ at the points of $\mathcal{E}_{r}$

For a suitably small $\varepsilon>0$ the touching points $\boldsymbol{r}\left(s_{i}(X)\right)$ of a tangent through the point

$$
X(x, y)=X_{0}+y \dot{\boldsymbol{r}}(\bar{s})+x \dot{\boldsymbol{r}}^{\perp}(\bar{s}), \quad \bar{s} \in\{a, b\}, \quad x, y \in(-\varepsilon, \varepsilon),
$$

depend continuously on $(x, y)$, hence $(6.3)$ leads to

$$
\begin{aligned}
f_{\bar{s}}(y) & :=\lim _{0<x \searrow 0}\left(\Delta M_{\boldsymbol{r}}(X(x, y))-\Delta M_{\boldsymbol{r}}(X(-x, y))\right) \\
& =\frac{\bar{\sigma} / \kappa(\bar{s})}{\left|y \dot{\boldsymbol{r}}(\bar{s})+X_{0}-\boldsymbol{r}(\bar{s})\right|^{3}}, \quad \text { where } \bar{\sigma} \in\{-1,+1\},
\end{aligned}
$$


because one of the existing touching points disappears either for $x$ or for $-x$ as Figure 6.2 shows.

Thus, one can determine the straight lines $\ell_{a}$ and $\ell_{b}$ by taking the segments in $\mathcal{R}$ on which $\Delta M_{\boldsymbol{r}}$ is discontinuous. Further, also the points $\boldsymbol{r}(a), \boldsymbol{r}(b)$ can be determined by considering $g(y):=1 / f_{\bar{s}}(y)$ on the segments $\mathcal{R} \cap \ell_{a}$ and $\mathcal{R} \cap \ell_{b}$, respectively, where $\bar{s}=a$ or $\bar{s}=b$, respectively. One only has to observe that $\pm \kappa(\bar{s})=g^{\prime \prime \prime}(0) / 6$ and $\left|X_{0}-\boldsymbol{r}(\bar{s})\right|=\left|g^{\prime \prime}(0)\right| / \kappa(\bar{s}) / 6$, and these together determine $\boldsymbol{r}(\bar{s})$.

Thus, from now on we know $\boldsymbol{r}(a), \boldsymbol{r}(b), \ell_{a}$ and $\ell_{b}$.

For the points $P \in \operatorname{Tr}\left(\left.\boldsymbol{r}\right|_{(a, b)}\right)$ there is an open domain $\mathcal{U} \subseteq \mathcal{R} \backslash \mathcal{E}_{\boldsymbol{r}}$ such that $\Delta M_{\boldsymbol{r}}$ is real analytic on $\mathcal{U}$ and there is an index $i$ in (6.3) such that $P=\boldsymbol{r}\left(s_{i}(X)\right)$ for some points $X \in \mathcal{U}$. Let $\widetilde{\Delta M_{\boldsymbol{r}}}$ be the unique maximal analytic continuation of $\left.\Delta M_{\boldsymbol{r}}\right|_{\mathcal{U}}$. Then by uniqueness we have

$$
\widetilde{\Delta M}_{\boldsymbol{r}}(X)=\sum_{i=1}^{2} \frac{1 / \tilde{\kappa}\left(\tilde{s}_{i}(X)\right)}{\left|X-\tilde{\boldsymbol{r}}\left(\tilde{s}_{i}(X)\right)\right|^{3}} \quad \text { or } \quad \widetilde{\Delta M}_{\boldsymbol{r}}(X)=\frac{1 / \tilde{\kappa}\left(\tilde{s}_{i}(X)\right)}{\left|X-\tilde{\boldsymbol{r}}\left(\tilde{s}_{i}(X)\right)\right|^{3}},
$$

where $\tilde{\kappa}$ is the curvature of $\tilde{\boldsymbol{r}}, \tilde{s}_{i}$ is the unique analytic extension of $s_{i} \mid \mathcal{U}$.

Let $\mathcal{M} \supseteq \mathcal{U}$ be the analyticity domain of $\widetilde{\Delta M}_{\boldsymbol{r}}$. As the curve $\tilde{\boldsymbol{r}}$ divides $\mathbb{R}^{2}$ into two connected open domains, the border of $\mathcal{M}$ is $\operatorname{Tr} \tilde{\boldsymbol{r}}$. Since $\tilde{\boldsymbol{r}}$ is an entire function of its arc-length parameter $s$, this determines $\tilde{\boldsymbol{r}}(\cdot+\hat{s})$, where the shift $\hat{s} \in \mathbb{R}$ is an unknown constant.

Thus, from now on we know $\tilde{\boldsymbol{r}}(\cdot+\hat{s})$ for an unknown shift $\hat{s} \in \mathbb{R}$.

Our next goal is to determine $\boldsymbol{r}(\cdot+\hat{s})=\left.\tilde{\boldsymbol{r}}(\cdot+\hat{s})\right|_{[a-\hat{s}, b-\hat{s}]}$.

If $\mathcal{K}$ is not bounded, then the points $\boldsymbol{r}(a)$ and $\boldsymbol{r}(b)$ split the curve $\tilde{\boldsymbol{r}}$ into three subcurves and only the middle one of these is bounded, hence $\boldsymbol{r}$ is that middle one.
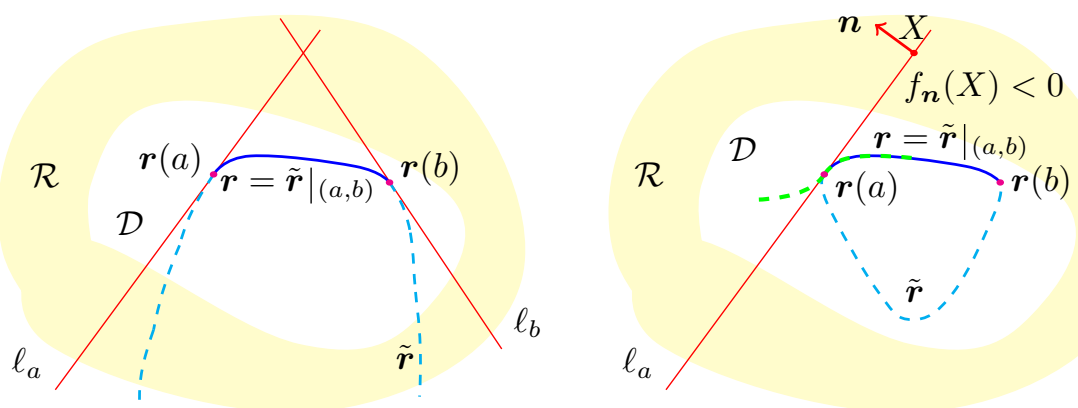

Figure 6.3. Determining $\boldsymbol{r}$ from $\tilde{\boldsymbol{r}}$ based on $\Delta M_{\boldsymbol{r}}$

If $\mathcal{K}$ is bounded, then the points $\boldsymbol{r}(a)$ and $\boldsymbol{r}(b)$ split curve $\tilde{\boldsymbol{r}}$ into two subcurves. Since we know $\operatorname{Tr} \tilde{\boldsymbol{r}}=\partial \mathcal{K}$, we know the side of $\ell_{a}$ on which $\mathcal{K}$ is placed. On the 
other hand, if $X \in \ell_{a} \cap \mathcal{R}$ and $\boldsymbol{n}$ is a normal unit vector of $\ell_{a}$, then (6.4) implies $f_{\boldsymbol{n}}(X):=\lim _{0<x \searrow 0}\left(\Delta M_{\boldsymbol{r}}(X+x \boldsymbol{n})-\Delta M_{\boldsymbol{r}}(X-x \boldsymbol{n})\right) \neq 0$. Moreover, as also Figure 6.3 shows, we have the following implications:

(i) if $f_{\boldsymbol{n}}(X)<0$, then for any point $P$ of $\operatorname{Tr} \boldsymbol{r}$ in a small neighbourhood of $\boldsymbol{r}(a)$ either $\boldsymbol{u}_{\boldsymbol{r}(a)}(P)$ or $\boldsymbol{u}_{P}(\boldsymbol{r}(a))$ is a positive linear combination of $\boldsymbol{n}$ and $\boldsymbol{u}_{X}(\boldsymbol{r}(a))$;

(ii) if $f_{\boldsymbol{n}}(X)>0$, then for any point $P$ of $\operatorname{Tr} \boldsymbol{r}$ in a small neighbourhood of $\boldsymbol{r}(a)$ either $\boldsymbol{u}_{\boldsymbol{r}(a)}(P)$ or $\boldsymbol{u}_{P}(\boldsymbol{r}(a))$ is a positive linear combination of $\boldsymbol{n}$ and $\boldsymbol{u}_{\boldsymbol{r}(a)}(X)$.

Thus, the curve $\boldsymbol{r}(\cdot+\hat{s})=\left.\tilde{\boldsymbol{r}}(\cdot+\hat{s})\right|_{[a-\hat{s}, b-\hat{s}]}$ is determined for an unknown shift $\hat{s} \in \mathbb{R}$.

Next, we consider the case of $\boldsymbol{r}(a)=\boldsymbol{r}(b), \ell_{a}=\ell_{b}$.

In this case, $\tilde{\boldsymbol{r}}$ has a period of length $b-a, \operatorname{Tr} \boldsymbol{r}=\partial \mathcal{K}$, hence $\mathcal{K} \subset \mathcal{D}$, and $\Delta M_{\boldsymbol{r}}$ is real analytic on $\mathcal{R}$. The unique maximal analytic continuation of $\left.\Delta M_{\boldsymbol{r}}\right|_{\mathcal{R}}$ is

$$
\widetilde{\Delta M}_{\boldsymbol{r}}(X)=\sum_{i=1}^{2} \frac{1 / \tilde{\kappa}\left(\tilde{s}_{i}(X)\right)}{\left|X-\tilde{\boldsymbol{r}}\left(\tilde{s}_{i}(X)\right)\right|^{3}} .
$$

The analyticity domain of $\widetilde{\Delta M}_{\boldsymbol{r}}$ is clearly the complement of $\mathcal{K}$, hence $\operatorname{Tr} \tilde{\boldsymbol{r}}=\partial \mathcal{K}$ determines $\tilde{\boldsymbol{r}}(\cdot+\hat{s})$, where the shift $\hat{s} \in \mathbb{R}$ is an unknown constant.

Thus, both cases above show that disregarding a shift in the arc-length parameter, the curve $\boldsymbol{r}$ is completely determined by $\Delta M_{\boldsymbol{r}}$.

So, for multicurves of one member curve, the theorem is proved. ${ }^{16}$

To complete the proof,

we consider now a multicurve $\boldsymbol{r}_{\mathcal{J}}$ of more member curves.

$\boldsymbol{r}_{j}:\left[a_{j}, b_{j}\right] \rightarrow \mathcal{D}$, where $j \in \mathcal{J}$. First we determine the set $\mathcal{L}_{\boldsymbol{r}_{\mathcal{J}}}$ of the tangent lines at the endpoints of $\boldsymbol{r}_{\mathcal{J}}$.

Let $\ell_{j, a}:=\ell\left(\boldsymbol{r}_{j}\left(a_{j}\right), \dot{\boldsymbol{r}}_{j}\left(a_{j}^{+}\right)\right), \ell_{j, b}:=\ell\left(\boldsymbol{r}_{j}\left(b_{j}\right), \dot{\boldsymbol{r}}_{j}\left(b_{j}^{-}\right)\right)$and $\mathcal{E}_{\boldsymbol{r}_{j}}:=\ell_{j, a} \cup \ell_{j, b}$. Define $\mathcal{E}_{\boldsymbol{r}_{\mathcal{J}}}:=\bigcup_{j \in \mathcal{J}} \mathcal{E}_{\boldsymbol{r}_{j}}$ and consider the set $\mathcal{E}_{\boldsymbol{r}_{\mathcal{J}}}^{\prime}$ of all the points in $\mathcal{R}$, where $\Delta M_{\boldsymbol{r}_{\mathcal{J}}}$ is not continuous. By (6.4), we have $\mathcal{E}_{\boldsymbol{r}_{\mathcal{J}}}^{\prime} \subseteq \mathcal{E}_{\boldsymbol{r}_{\mathcal{J}}} \cap \mathcal{R}$, and we show now that the closure of the set $\mathcal{E}_{\boldsymbol{r}_{\mathcal{J}}}^{\prime}$ is $\mathcal{R} \cap \mathcal{E}_{\boldsymbol{r}_{\mathcal{J}}}$.

Let $X_{0} \in \mathcal{R}$ be on a straight line $\ell \in \mathcal{E}_{\boldsymbol{r}_{\mathcal{J}}}$ of normal vector $\boldsymbol{n}$. Assume that no other straight line in $\mathcal{E}_{\boldsymbol{r}_{\mathcal{J}}}$ passes through $X_{0}$. Then there is a small $\varepsilon>0$ such that the points

$$
X(x, y)=X_{0}+x \boldsymbol{n}+y \boldsymbol{n}^{\perp}, \quad x, y \in(-\varepsilon, \varepsilon)
$$

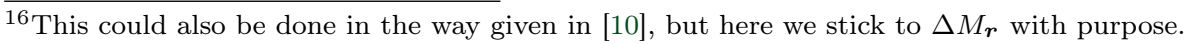


are in $\mathcal{R}$ and not in $\mathcal{E}_{\boldsymbol{r}_{\mathcal{J}}}$ except $\ell$. Assume that $\ell=\ell\left(\boldsymbol{r}_{j_{1}}\left(\bar{s}_{j_{1}}\right), \dot{\boldsymbol{r}}_{j_{1}}\left(\bar{s}_{j_{1}}^{ \pm}\right)\right)=\cdots=$ $\ell\left(\boldsymbol{r}_{j_{k}}\left(\bar{s}_{j_{k}}\right), \dot{\boldsymbol{r}}_{j_{k}}\left(\bar{s}_{j_{k}}^{ \pm}\right)\right)$, where $j_{1}, \ldots, j_{k} \in \mathcal{J}$,

$$
\bar{s}_{j_{i}} \in\left\{a_{j_{i}}, b_{j_{i}}\right\}, \quad \text { and } \bar{s}_{j_{i}}^{ \pm}=\left\{\begin{array}{ll}
a_{j_{i}}^{+}, & \text {if } \bar{s}_{j_{i}}=a_{j_{i}}, \\
b_{j_{i}}^{-}, & \text {if } \bar{s}_{j_{i}}=b_{j_{i}},
\end{array} \quad(i=1, \ldots, k) .\right.
$$

Then (6.4) implies that

$$
\begin{aligned}
f_{X_{0}, \ell}(y) & :=\lim _{0<x \searrow 0}\left(\Delta M_{\boldsymbol{r}_{\mathcal{J}}}(X(x, y))-\Delta M_{\boldsymbol{r}_{\mathcal{J}}}(X(-x, y))\right) \\
& =\sum_{i=1}^{k} \frac{\bar{\sigma}_{j_{i}} / \kappa_{j_{i}}\left(\bar{s}_{j_{i}}^{ \pm}\right)}{\left|y \boldsymbol{n}^{\perp}+X_{0}-\boldsymbol{r}_{j_{i}}\left(\bar{s}_{j_{i}}\right)\right|^{3}}, \quad \text { where } \bar{\sigma}_{j_{i}} \in\{-1,+1\} .
\end{aligned}
$$

If $f_{X_{0}, \ell}$ vanishes on an interval, then Theorem A.1 implies that $k$ is even, $\kappa_{j_{i}}\left(\bar{s}_{j_{i}}^{ \pm}\right)=$ $\kappa_{j_{k+1-i}}\left(\bar{s}_{j_{k+1-i}}^{ \pm}\right)$and $\boldsymbol{r}_{j_{i}}\left(\bar{s}_{j_{i}}\right)=\boldsymbol{r}_{j_{k+1-i}}\left(\bar{s}_{j_{k+1-i}}\right)$ with a suitable indexing. This contradicts the tangency condition in the theorem, hence $\Delta M_{\boldsymbol{r}_{\mathcal{J}}}$ is discontinuous in an everywhere dense subset of the segment $\ell \cap \mathcal{R}$, which was to be proved.

Thus, we have determined the set $\mathcal{L}_{\boldsymbol{r}_{\mathcal{J}}}$ of the straight lines of the form $\ell\left(\boldsymbol{r}_{j}\left(\bar{s}_{j}\right), \dot{\boldsymbol{r}}_{j}\left(\bar{s}_{j}^{ \pm}\right)\right)$. Further, using Theorem A.1 on formula (6.6) for every straight line in $\mathcal{L}_{\boldsymbol{r}_{\mathcal{J}}}$, we get the triplets $\left(\boldsymbol{r}_{j_{i}}\left(\bar{s}_{j_{i}}\right), \ell_{\bar{s}_{j_{i}}}, \kappa\left(\bar{s}_{j_{i}}^{ \pm}\right)\right)$for every endpoint of the curves in $\boldsymbol{r}_{\mathcal{J}}$.

Next, we determine (up to an unknown shift) the analytic extensions $\tilde{\boldsymbol{r}}_{j}$ of some curves $\boldsymbol{r}_{j}$.

Let $\mathcal{U} \subseteq \mathcal{R}$ be an open domain on which $\Delta M_{\boldsymbol{r}_{\mathcal{J}}}$ is real analytic. By (6.5) we have

$$
\Delta M_{\boldsymbol{r}_{\mathcal{J}}}(X)=\sum_{j \in \mathcal{J}} \sum_{i=1}^{m_{j}} \frac{1 / \kappa_{j}\left(s_{j, i}(X)\right)}{\left|X-\boldsymbol{r}_{j}\left(s_{j, i}(X)\right)\right|^{3}} \text { for } X \in \mathcal{U},
$$

where the otherwise usual notations indexed additionally by $j$ to express their belonging to the member curve $\boldsymbol{r}_{j}$, and $m_{j} \in\{0,1,2\} .{ }^{17}$ Then, by uniqueness, the maximal analytic continuation of $\Delta M_{\boldsymbol{r}_{\mathcal{J}}} \mid \mathcal{U}$ is

$$
\widetilde{\Delta M}_{\boldsymbol{r}_{\mathcal{J}}}(X)=\sum_{j \in \mathcal{J}} \sum_{i=1}^{m_{j}} \frac{1 / \tilde{\kappa}_{j}\left(\tilde{s}_{j, i}(X)\right)}{\left|X-\tilde{\boldsymbol{r}}_{j}\left(\tilde{s}_{j, i}(X)\right)\right|^{3}},
$$

where $\tilde{s}_{j, i}$ is the unique analytic extension of $s_{j, i} \mid \mathcal{U}$.

Consider now the maximal domain $\mathcal{M} \supseteq \mathcal{U}$ on which $\widetilde{\Delta M}_{\boldsymbol{r}_{\mathcal{J}}}$ is analytic. As the curves $\tilde{\boldsymbol{r}}_{j}(j \in \mathcal{J})$ divide $\mathbb{R}^{2}$ into finitely many connected open domains, $\mathcal{M}$ is that one of these domains that contains $\mathcal{U}$ as a subset. (See Figure 6.4.)

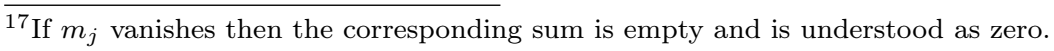




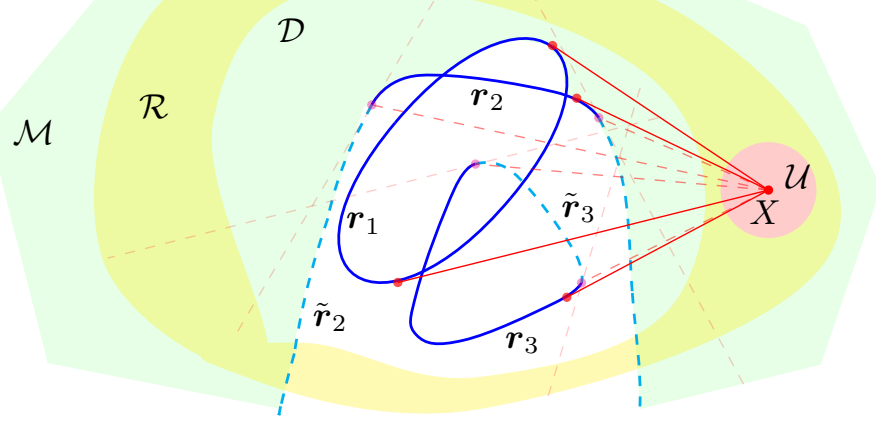

Figure 6.4. The analyticity domain $\mathcal{M}$ is bordered by $\boldsymbol{r}_{1}$ and $\boldsymbol{r}_{2}$

As such, the border $\partial \mathcal{M}$ of $\mathcal{M}$ is the union of the traces of some subcurves of $\tilde{\boldsymbol{r}}_{\bar{\jmath}}$ for certain set $\overline{\mathcal{J}}$ of indexes $\bar{\jmath} \in \mathcal{J}$. Since every $\tilde{\boldsymbol{r}}_{\bar{\jmath}}$ is an entire function of its arc-length parameter, any arc in $\partial \mathcal{M}$ determines a curve $\tilde{\boldsymbol{r}}_{\bar{\jmath}}\left(\cdot+\hat{s}_{\bar{\jmath}}\right)$, where the shift $\hat{s}_{\bar{\jmath}} \in \mathbb{R}$ is an unknown constant.

To determine member curves $\boldsymbol{r}_{j}$ of $\boldsymbol{r}_{\mathcal{J}}$ that are subcurves of the already known analytic curve $\tilde{\boldsymbol{r}}_{\bar{\jmath}}\left(\cdot+\hat{s}_{\bar{\jmath}}\right)$ (the shift $\hat{s}_{\bar{\jmath}}$ is unknown), we first match the triplets $\left(\boldsymbol{r}_{j}\left(\bar{s}_{j}^{ \pm}\right), \ell_{\bar{s}_{j}^{ \pm}}, \kappa\left(\bar{s}_{j}^{ \pm}\right)\right)$, known at the endpoints of the member curves, with the triplets of the form $\left(\tilde{\boldsymbol{r}}_{\bar{\jmath}}(s), \ell\left(\tilde{\boldsymbol{r}}_{\bar{\jmath}}(s), \tilde{\boldsymbol{r}}_{\bar{\jmath}}^{\prime}(s)\right), \tilde{\kappa}_{\bar{\jmath}}(s)\right)$, known for every $s \in \mathbb{R}$. The number of matches is clearly an even number $n_{\bar{\jmath}}$, because no two member curves are tangent at order two.

If $n_{\bar{\jmath}}=0$, then no endpoint of a member curve of $\boldsymbol{r}_{\mathcal{J}}$ is on $\operatorname{Tr} \tilde{\boldsymbol{r}}_{\bar{\jmath}}$, therefore $\tilde{\boldsymbol{r}}_{\bar{\jmath}}$ is periodic, and it is a member curve of $\boldsymbol{r}_{\mathcal{J}}$.

If $n_{\bar{\jmath}}=2 k_{\bar{\jmath}}$, where $k_{\bar{\jmath}} \geq 1$, then we obtain

$$
\begin{aligned}
\left.\left(\tilde{\boldsymbol{r}}_{\bar{\jmath}}\left(\bar{s}_{i}\right), \ell\left(\tilde{\boldsymbol{r}}_{\bar{\jmath}}\left(\bar{s}_{i}\right), \tilde{\boldsymbol{r}}_{\bar{\jmath}}^{\prime}\left(\bar{s}_{i}\right)\right), \tilde{\kappa}_{\bar{\jmath}}\left(\bar{s}_{i}\right)\right)\right) & =\left(\boldsymbol{r}_{j_{i}}\left(a_{j_{i}}\right), \ell_{a_{j_{i}}}, \kappa\left(a_{j_{i}}^{+}\right)\right), \\
\left.\left(\tilde{\boldsymbol{r}}_{\bar{\jmath}}\left(\bar{s}_{2 i}\right), \ell\left(\tilde{\boldsymbol{r}}_{\bar{\jmath}}\left(\bar{s}_{2 i}\right), \tilde{\boldsymbol{r}}_{\bar{\jmath}}^{\prime}(2 i)\right), \tilde{\kappa}_{\bar{\jmath}}\left(\bar{s}_{2 i}\right)\right)\right) & =\left(\boldsymbol{r}_{\bar{\jmath}}\left(b_{j_{i}}\right), \ell_{b_{j_{i}}}, \kappa\left(b_{j_{i}}^{-}\right)\right)
\end{aligned}
$$

for suitable $\bar{s}_{i}$ and $\bar{s}_{2 i}$, where $i=1, \ldots, k_{\bar{\jmath}}$. Let $\mathcal{K}_{\bar{\jmath}}$ be the convex domain for which $\operatorname{Tr} \tilde{\boldsymbol{r}}_{\bar{\jmath}}=\partial \mathcal{K}_{\bar{\jmath}}$.

If $\mathcal{K}_{\bar{\jmath}}$ is not bounded, then the points $\tilde{\boldsymbol{r}}_{\bar{\jmath}}\left(\bar{s}_{i}\right)\left(i=1, \ldots, 2 k_{\bar{\jmath}}\right)$ split the curve $\tilde{\boldsymbol{r}}_{\bar{\jmath}}$ into $2 k_{\bar{\jmath}}+1$ subcurves two of which are unbounded. Assume that the points $\tilde{\boldsymbol{r}}_{\bar{\jmath}}\left(\bar{s}_{i_{m}}\right)$ are placed on $\operatorname{Tr} \tilde{\boldsymbol{r}}_{\bar{\jmath}}=\partial \mathcal{K}_{\bar{\jmath}}$ so that $\tilde{\boldsymbol{r}}_{\bar{\jmath}}\left(\bar{s}_{i_{1}}\right)$ and $\tilde{\boldsymbol{r}}_{\bar{\jmath}}\left(\bar{s}_{i_{2 k_{\bar{j}}}}\right)$ are the endpoints of the two unbounded subcurves, respectively, and $\bar{s}_{i_{1}}, \ldots, \bar{s}_{i_{m}}, \ldots, \bar{s}_{i_{2 k_{j}}}$ is a monotonously ascending or descending sequence. Then the member curves of $\boldsymbol{r}_{\mathcal{J}}$ on $\tilde{\boldsymbol{r}}_{\bar{\jmath}}$ are $\tilde{\boldsymbol{r}}_{\bar{\jmath}} \mid\left[\bar{s}_{i_{2 m+1}}, \ldots, \bar{s}_{i_{2 m+2}}\right]$, where $m=1, \ldots, k_{\bar{\jmath}}$. 
If $\mathcal{K}_{\bar{\jmath}}$ is bounded, then the points $\tilde{\boldsymbol{r}}_{\bar{\jmath}}\left(\bar{s}_{i}\right)\left(i=1, \ldots, 2 k_{\bar{\jmath}}\right)$ split the curve $\tilde{\boldsymbol{r}}_{\bar{\jmath}}$ into $2 k_{\bar{\jmath}}$ subcurves (all of which are bounded). Assume that the points $\tilde{\boldsymbol{r}}_{\bar{\jmath}}\left(\bar{s}_{i_{m}}\right)$ are placed on $\operatorname{Tr} \tilde{\boldsymbol{r}}_{\bar{\jmath}}=\partial \mathcal{K}_{\bar{\jmath}}$ so that $\tilde{\boldsymbol{r}}_{\bar{\jmath}}\left(\bar{s}_{i_{1}}\right), \ldots, \tilde{\boldsymbol{r}}_{\bar{\jmath}}\left(\bar{s}_{i_{m}}\right), \ldots, \tilde{\boldsymbol{r}}_{\bar{j}}\left(\bar{s}_{i_{2 k_{\bar{j}}}}\right)$ is a sequence of points arranged anticlockwise. As $\operatorname{Tr} \tilde{\boldsymbol{r}}_{\bar{\jmath}}=\partial \mathcal{K}_{\bar{\jmath}}$ is known, the side of $\ell\left(\tilde{\boldsymbol{r}}_{\bar{\jmath}}\left(\bar{s}_{i_{1}}\right), \tilde{\boldsymbol{r}}_{\bar{\jmath}}^{\prime}\left(\bar{s}_{i_{1}}\right)\right)$ on which the domain $\mathcal{K}_{\bar{\jmath}}$ is placed is known, hence observing equation (6.4) and figure 6.1 one can deduce for the member curve $\boldsymbol{r}$ on $\partial \mathcal{K}_{\bar{\jmath}}$ ending at $\tilde{\boldsymbol{r}}_{\bar{\jmath}}\left(\bar{s}_{i_{1}}\right)$ that

(i) if $\bar{\sigma}<0$, then for any point $P$ of $\operatorname{Tr} \boldsymbol{r}$ in a small neighbourhood of $\tilde{\boldsymbol{r}}_{\bar{\jmath}}\left(\bar{s}_{i_{1}}\right)$ either $\boldsymbol{u}_{\tilde{\boldsymbol{r}}_{\bar{\jmath}}\left(\bar{s}_{i_{1}}\right)}(P)$ or $\boldsymbol{u}_{P}\left(\tilde{\boldsymbol{r}}_{\bar{\jmath}}\left(\bar{s}_{i_{1}}\right)\right)$ is a positive linear combination of $\dot{\boldsymbol{r}}_{\bar{\jmath}}^{\perp}\left(\bar{s}_{i_{1}}\right)$ and $\boldsymbol{u}_{X}\left(\tilde{\boldsymbol{r}}_{\bar{\jmath}}\left(\bar{s}_{i_{1}}\right)\right)$;

(ii) if $\bar{\sigma}>0$, then for any point $P$ of $\operatorname{Tr} \boldsymbol{r}$ in a small neighbourhood of $\tilde{\boldsymbol{r}}_{\bar{\jmath}}\left(\bar{s}_{i_{1}}\right)$ either $\boldsymbol{u}_{\tilde{\boldsymbol{r}}_{\bar{\jmath}}\left(\bar{s}_{i_{1}}\right)}(P)$ or $\boldsymbol{u}_{P}\left(\tilde{\boldsymbol{r}}_{\bar{\jmath}}\left(\bar{s}_{i_{1}}\right)\right)$ is a positive linear combination of $\dot{\boldsymbol{r}}_{\bar{\jmath}}^{\perp}\left(\bar{s}_{i_{1}}\right)$ and $-\boldsymbol{u}_{X}\left(\tilde{\boldsymbol{r}}_{\bar{\jmath}}\left(\bar{s}_{i_{1}}\right)\right)$.

This determines the member curve $\boldsymbol{r}$ on $\partial \mathcal{K}_{\bar{\jmath}}$ that is ending at $\tilde{\boldsymbol{r}}_{\bar{\jmath}}\left(\bar{s}_{i_{1}}\right)$. It is either

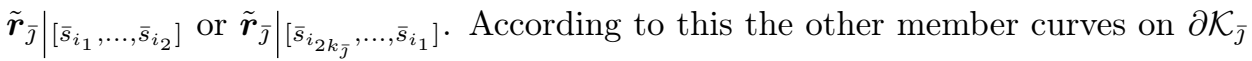
are either $\left.\tilde{\boldsymbol{r}}_{\bar{\jmath}}\right|_{\left[\bar{s}_{i_{2 m+1}}, \ldots, \bar{s}_{i_{2 m+2}}\right]}$ or $\left.\tilde{\boldsymbol{r}}_{\bar{\jmath}}\right|_{\left.\bar{s}_{i_{2 m}}, \ldots, \bar{s}_{i_{2 m+1}}\right]}$, respectively, for $m=1, \ldots, k_{\bar{\jmath}}-1$.

Thus, disregarding shifts in their arc-length parameter, all member curves on $\partial \mathcal{K}_{\bar{\jmath}}$ are completely determined by $\Delta M_{\boldsymbol{r}_{\mathcal{J}}}$. Let $\overline{\mathcal{J}}$ be the set of the indexes of these member curves, and observe that $\Delta M_{\boldsymbol{r}_{\mathcal{J}}}-\Delta M_{\boldsymbol{r}_{\overline{\mathcal{J}}}}=\Delta M_{\boldsymbol{r}_{\mathcal{J}^{\prime}}}$, where $\mathcal{J}^{\prime}=\mathcal{J} \backslash \overline{\mathcal{J}}$, is now known. So, taking $\Delta M_{\boldsymbol{r}_{\mathcal{J}^{\prime}}}$ into consideration, one can again determine the non-empty set of member curves of $\boldsymbol{r}_{\mathcal{J}^{\prime}}$ on a due analytic curve, say the set of the indexes of these is $\hat{\mathcal{J}}$, allowing the definition of $\mathcal{J}^{\prime \prime}=\mathcal{J}^{\prime} \backslash \mathcal{J}$ in which case $\Delta M_{\boldsymbol{r}_{\mathcal{J}^{\prime}}}-\Delta M_{\boldsymbol{r}_{\hat{\mathcal{J}}}}=\Delta M_{\boldsymbol{r}_{\mathcal{J}^{\prime}}}$. Repeating this procedure determines at least one new member curve of $\boldsymbol{r}_{\mathcal{J}}$ in every step.

As $\mathcal{J}$ is finite, this proves the theorem completely.

Theorem 6.2 sharpens [2, Theorem 10.2] and [16, Theorem 2.1] for a somewhat smaller class of multicurves by requesting the coincidence of the masking functions in a ring only.

Exclusion of points of undulation and inflection can not be easily dropped from Theorem 6.2, because $\Delta M_{\boldsymbol{r}_{\mathcal{J}}}$ tends to infinity at the straight lines touching the multicurve at a point of vanishing curvature. See Figure 6.5 for an extreme situation, where the analytic extensions of $\left.\Delta M_{\left\{\boldsymbol{r}_{1}, \boldsymbol{r}_{2}\right\}}\right|_{\mathcal{R}}$ can not reach the multicurve $\left\{\boldsymbol{r}_{1}, \boldsymbol{r}_{2}\right\}$, because the straight lines touching the multicurve in points of vanishing curvature block the extension. 


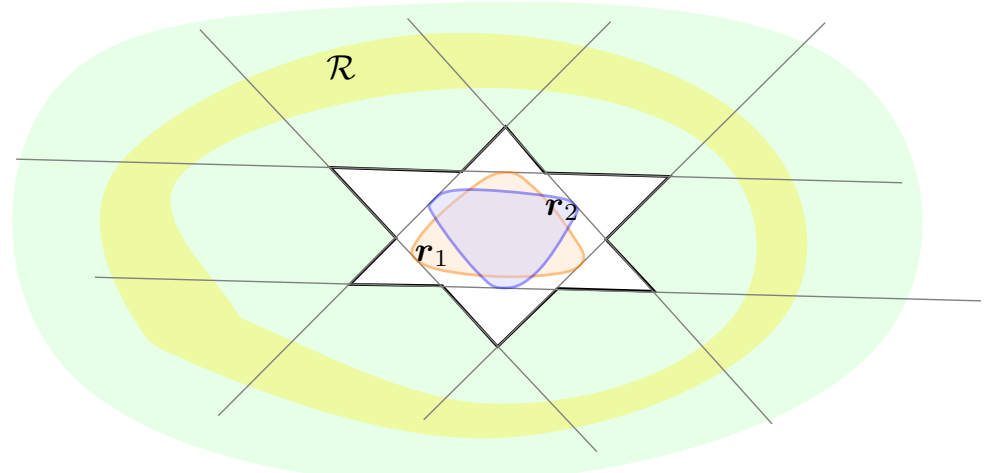

Figure 6.5. The analytic extensions of $\left.\Delta M_{\left\{\boldsymbol{r}_{1}, \boldsymbol{r}_{2}\right\}}\right|_{\mathcal{R}}$ can not reach the multicurve $\left\{\boldsymbol{r}_{1}, \boldsymbol{r}_{2}\right\}$.

\section{Discussion}

The following consequence of Theorem 6.2 is similar in spirit to J. C. C. Nitsche's result ${ }^{18}$ in [15].

Corollary 7.1. Let $\mathcal{R}$ be an open ring bounded by two concentric circles of radius a and $b>a$ centred to the origin. Let the multicurve $\boldsymbol{r}_{\mathcal{J}} \in \mathfrak{E}$ be inside the disc $\mathcal{D}_{a}$ of radius a centred to the origin such that no two of its member curves are tangent of order two at any of their endpoints. If the masking function $M_{\boldsymbol{r}_{\mathcal{J}}}$ is constant on every circle of radius $\varrho \in(a, b)$ centred to the origin, then every member curve of $\boldsymbol{r}_{\mathcal{J}}$ is a circle centred to the origin with radius less than a.

The proof is simple: by Theorem 6.2 the restriction $\left.\bar{M}_{\boldsymbol{r}_{\mathcal{J}}}\right|_{\mathcal{R}}$ uniquely determines $\operatorname{Tr} \boldsymbol{r}_{\mathcal{J}}$, therefore $\boldsymbol{r}_{\mathcal{J}}$ should have the same rotational symmetry as $\left.\bar{M}_{\boldsymbol{r}_{\mathcal{J}}}\right|_{\mathcal{R}}$ has. This result raises the following question:

(q) Is it enough to know that $M_{\boldsymbol{r}_{\mathcal{J}}}$ is constant on finitely many concentric circles to determine $\boldsymbol{r}_{\mathcal{J}}$ if an a priori upper limit for $|\mathcal{J}|$ would be given in Corollary 7.1? Nitsche's result in [15] answers this question affirmatively for $|\mathcal{J}|=1$. Corollary 7.1 says that countable many concentric circles are always enough.

We generalize the notion of the masking function to higher dimensions as follows. Let $\mathcal{L}$ be the Grassman manifold of the straight lines in $\mathbb{R}^{n}(n \geq 2)$, and let $\bar{\omega}: \mathcal{L} \rightarrow \mathbb{R}_{+}$be a strictly positive continuous function called also line weight. The $k$-dimensional $(2 \leq k \leq n)$ weighted masking number $M_{\mathcal{T} ; \bar{\omega}}(P)$ of the trace

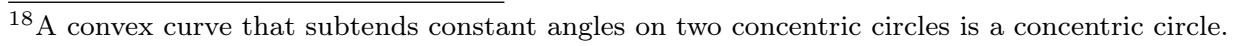


$\mathcal{T}=\operatorname{Tr} \boldsymbol{r}_{\mathcal{J}}$ of the multisurface ${ }^{19} \boldsymbol{r}_{\mathcal{J}}$ is defined by

$$
M_{\mathcal{T} ; \bar{\omega}}(P, \hbar)=\frac{1}{2} \int_{\mathbb{S}^{n-1} \cap \hbar} \#(\mathcal{T} \cap \ell(P, \boldsymbol{w})) \bar{\omega}(\ell(P, \boldsymbol{w})) d \boldsymbol{w},
$$

where $\mathbb{S}^{n-1}$ is the unit sphere, $\hbar$ is a $k$-dimensional subspace of $\mathbb{R}^{n}, \ell(P, \boldsymbol{w})$ is the straight line through the point $P \in \mathbb{R}^{n}$ with direction $\boldsymbol{w} \in \mathbb{S}^{n-1} \cap \hbar$ and \# is the counting measure. We call the point $P$, the subspace $\hbar$ and the set $\mathcal{T}$ the source, the field of view and the object, respectively, of the masking number $M_{\mathcal{T} ; \bar{\omega}}(P, \hbar)$. The function $M_{\mathcal{T}: \bar{\omega}}: \mathbb{R}^{n} \times \mathcal{G}^{n, k} \rightarrow \mathbb{R}_{0 \leq}$, where $\mathcal{G}^{n, k}$ is the Grassman manifold of the $k$-dimensional subspaces of $\mathbb{R}^{n}$, is called the weighted masking function of $\mathcal{T}$.

Let $k<n$. Then $M_{\mathcal{T}: \bar{\omega}}(P, \hbar)$ is the $k$-dimensional Radon transform of the function $\#(\mathcal{T} \cap \ell(P, \boldsymbol{w})) \bar{\omega}(\ell(P, \boldsymbol{w}))$ on the sphere $\mathbb{S}_{P}^{n-1}$ centred at the point $P$. This Radon transform is invertible [18], therefore its inverse gives back the function $\#(\mathcal{T} \cap \ell(P, \boldsymbol{w})) \bar{\omega}(\ell(P, \boldsymbol{w}))$ for every $\boldsymbol{w} \in \mathbb{S}^{n-1}$.

Theorem 7.2. Let $\mathcal{D} \subset \mathbb{R}^{n}$ be a compact domain and let $1<k<n$. Let the trace of multisurface $\boldsymbol{r}_{\mathcal{J}}$ be in $\mathcal{D}$, and assume that the intersection of $\boldsymbol{r}_{\mathcal{J}}$ with any 2dimensional plane is a rectifiable multicurve. Then the trace of the multisurface $\boldsymbol{r}_{\mathcal{J}}$ can be determined by knowing its $k$-dimensional weighted masking function $\bar{M}_{\boldsymbol{r}_{\mathcal{J}}}$ at every point on $\partial \mathcal{D}$.

The task is to determine for every 2-dimensional plane $\mathcal{P}$ the rectifiable multicurve $\boldsymbol{p}=\operatorname{Tr} \boldsymbol{r}_{\mathcal{J}} \cap \mathcal{P}$. By the observations made before the theorem, we have $\#(\operatorname{Tr} \boldsymbol{p} \cap \ell)$ for every straight line $\ell \subset \mathcal{P}$. The results in $[2,16,17]$ prove that this is enough to determine $\boldsymbol{p}$, hence Theorem 7.2 is proved. If $k=n$, the method of this proof can not be used.

For $k=n>2$ the only positive result known by the author is the following.

Theorem. ([14, Theorem 5.1]) Let $\varrho_{2}>\varrho_{1}>\bar{r}>0$ and let $\mathcal{K} \subset \mathbb{R}^{n}(n \neq 3)$ be a convex body contained in the interior of the ball $\varrho_{1} \mathcal{B}^{n}$. If the masking functions of $\partial \mathcal{K}$ and $\bar{r} \mathcal{B}$ are equal on $\varrho_{1} \mathbb{S}^{n-1}$ and on $\varrho_{2} \mathbb{S}^{n-1}$, then $\mathcal{K}=\bar{r} \mathcal{B}$.

\section{A. Appendix}

Theorem A.1. Assume that the function $f:(a, b) \rightarrow \mathbb{R} \cup\{\infty,-\infty\}$ can be written in the form $f(x)=p(x)+\sum_{i=1}^{k} \frac{c_{i}}{\left(x-d_{i}\right)^{e_{i}}}$ for a polynomial $p$ and some $k, e_{i} \in \mathbb{N}$ and $c_{i}, d_{i} \in \mathbb{R}$ such that $d_{i} \neq d_{j}$ for every $i, j=1, \ldots, k$. Then the set $\{p\} \cup$ $\left\{\left(c_{i}, d_{i}, e_{i}\right)\right\}_{i=1}^{k}$ is unique.

${ }^{19} \mathrm{~A}$ multisurface is a finite set of regular surfaces of duly prescribed properties. 
Proof. As $f$ can only be infinite at finitely many arguments, we can find a subinterval $\mathbb{I} \subset(a, b)$ on which $f$ is bounded. Let $g_{0}$ be the restriction of $f$ onto $\mathbb{I}$, and choose a number $x_{0} \in \mathbb{I}$. Then $g_{0}$ can be written in the form

$$
g_{0}(x)=p(x)+\sum_{i=1}^{k} \frac{-c_{i} /\left(d_{i}-x_{0}\right)^{e_{i}}}{\left(1-\left(x-x_{0}\right) /\left(d_{i}-x_{0}\right)\right)^{e_{i}}},
$$

where for convenience we assume

$$
\left|d_{1}-x_{0}\right| \leq\left|d_{2}-x_{0}\right|<\left|d_{3}-x_{0}\right| \leq \cdots \leq\left|d_{i}-x_{0}\right| \leq \cdots \leq\left|d_{k}-x_{0}\right| .
$$

The $m$ th $(m \in \mathbb{N})$ derivatives of $g_{0}$ at $x_{0}$ are

$$
g_{0}^{(m)}\left(x_{0}\right)=p^{(m)}(x)+(-1)^{m+1} \sum_{i=1}^{k} \frac{\left(m+e_{i}-1\right) !}{\left(e_{i}-1\right) !} \frac{c_{i}}{\left(d_{i}-x_{0}\right)^{m+e_{i}}},
$$

hence for any $j \in\{0,1\}$ and $m_{j}(n)=2 n+1+j$ we have

$$
\begin{aligned}
& h_{j}\left(x_{0}\right) \\
& :=\lim _{n \rightarrow \infty} \sqrt[2 n+1]{\frac{g_{0}^{\left(m_{j}(n)\right)}\left(x_{0}\right)}{\left(m_{j}(n)\right) !(-1)^{j}}} \\
& =\lim _{n \rightarrow \infty}\left(\sum_{i=1}^{k}\left(\begin{array}{c}
m_{j}(n)+e_{i}-1 \\
e_{i}-1
\end{array}\right) \frac{c_{i}}{\left(d_{i}-x_{0}\right)^{m_{j}(n)+e_{i}}}\right)^{1 /(2 n+1)} \\
& =\frac{1}{d_{1}-x_{0}} \lim _{n \rightarrow \infty}\left(\frac{p_{1}(n) c_{1}}{\left(d_{1}-x_{0}\right)^{j+e_{1}}}+\sum_{i=2}^{k} \frac{p_{i}(n) c_{i}}{\left(d_{i}-x_{0}\right)^{j+e_{i}}}\left(\frac{d_{1}-x_{0}}{d_{i}-x_{0}}\right)^{2 n+1}\right)^{1 /(2 n+1)}
\end{aligned}
$$

where every $p_{i}$ is a polynomial of degree $e_{i}$ with positive leading coefficient. It is easy to see now that

$$
\begin{aligned}
h_{j}\left(x_{0}\right) & =\frac{1}{d_{1}-x_{0}} \lim _{n \rightarrow \infty}\left(\frac{p_{1}(n) c_{1}}{\left(d_{1}-x_{0}\right)^{j+e_{1}}}+\frac{p_{2}(n) c_{2}}{\left(d_{2}-x_{0}\right)^{j+e_{2}}}\left(\frac{d_{1}-x_{0}}{d_{2}-x_{0}}\right)^{2 n+1}\right)^{1 /(2 n+1)} \\
& =\frac{1}{d_{1}-x_{0}} \begin{cases}\frac{\operatorname{sign} c_{1}}{\operatorname{sign}\left(\left(d_{1}-x_{0}\right)^{j+e_{1}}\right)}, & \text { if }\left|d_{1}-x_{0}\right|<\left|d_{2}-x_{0}\right|, \\
\frac{\operatorname{sign} c_{1}}{\operatorname{sign}\left(\left(d_{1}-x_{0}\right)^{\left.j+e_{1}\right)}\right.}, & \text { if } d_{1}-x_{0}=x_{0}-d_{2} \text { and } e_{1}>e_{2}, \\
\frac{-\operatorname{sign} c_{2}}{\operatorname{sign}\left(\left(d_{2}-x_{0}\right)^{\left.j+e_{2}\right)},\right.}, & \text { if } d_{1}-x_{0}=x_{0}-d_{2} \text { and } e_{1}<e_{2}, \\
\frac{\operatorname{sign}\left(c_{1}-(-1)^{j+e_{2}} c_{2}\right)}{\operatorname{sign}\left(\left(d_{1}-x_{0}\right)^{j+e_{1}}\right)}, & \text { if } d_{1}-x_{0}=x_{0}-d_{2} \text { and } e_{1}=e_{2} .\end{cases}
\end{aligned}
$$

This means that either $h_{0}\left(x_{0}\right) \neq 0$ or $h_{1}\left(x_{0}\right) \neq 0$. If $h_{j}\left(x_{0}\right) \neq 0$, then $\left|d_{1}-x\right|=$ $1 /\left|h_{j}(x)\right|$ for every $x$ in a small neighbourhood of $x_{0}$, thus $d_{1}$ can be determined easily. 
Having $d_{1}$, let $\varepsilon>0$ be such that $x_{1}=x_{0}+\varepsilon\left(d_{1}-x_{0}\right)$ is in $\mathbb{I}$. Then we have

$$
\begin{aligned}
& \lim _{n \rightarrow \infty} \log _{m_{j}(n)}\left(\frac{g_{0}^{\left(m_{j}(n)\right)}\left(x_{1}\right)\left(d_{1}-x_{1}\right)^{2 n+1}}{\left(m_{j}(n)\right) !(-1)^{j}}\right) \\
& \quad=\lim _{n \rightarrow \infty} \log _{m_{j}(n)}\left(\frac{p_{1}(n) c_{1}}{\left(d_{1}-x_{1}\right)^{j+e_{1}}}+\sum_{i=2}^{k} \frac{p_{i}(n) c_{i}}{\left(d_{i}-x_{1}\right)^{j+e_{i}}}\left(\frac{d_{1}-x_{1}}{d_{i}-x_{1}}\right)^{2 n+1}\right) \\
& \quad=\lim _{n \rightarrow \infty} \log _{m_{j}(n)}\left(\left(\begin{array}{c}
m_{j}(n)+e_{1}-1 \\
e_{1}-1
\end{array}\right) \frac{c_{1}}{\left(d_{1}-x_{1}\right)^{j+e_{1}}}\right) \\
& =e_{1}-1 .
\end{aligned}
$$

Having $d_{1}$ and $e_{1}$ we obtain

$$
\lim _{n \rightarrow \infty} \frac{g_{0}^{\left(m_{j}(n)\right)}\left(x_{1}\right)\left(d_{1}-x_{1}\right)^{m_{j}(n)+e_{1}}}{\left(m_{j}(n)\right) !(-1)^{j}\left(\begin{array}{c}
m_{j}(n)+e_{1}-1 \\
e_{1}-1
\end{array}\right)}=c_{1} .
$$

Thus, having $g_{0}$ we determined $d_{1}, c_{1}$ and $e_{1}$.

By induction, assume that we already know the values $c_{1}, \ldots, c_{j}, d_{1}, \ldots, d_{j}$, $e_{1}, \ldots, e_{j}$ and the functions $g_{0}, \ldots, g_{j-1}$ for some $0<j<k$. Then the function

$g_{j}: \mathbb{I} \ni x \mapsto g_{j-1}(x)-\frac{c_{j}}{\left(x-d_{j}\right)^{e_{j}}} \in \mathbb{R}$ satisfies every condition of the theorem, it is bounded on $\mathbb{I}$, and therefore the above method produces $d_{j+1}, c_{j+1}$ and $e_{j+1}$. Repeating this procedure until $g_{k+1}=0$, we find the values $d_{1}, \ldots, d_{k}, c_{1}, \ldots, c_{k}$ and $e_{1}, \ldots, e_{k}$.

Finally, knowing $p: x \mapsto f(x)-\sum_{i=1}^{k} \frac{c_{i}}{\left(x-d_{i}\right)^{e_{i}}}$ on the interval $\mathbb{I}$, determine the polynomial $p$ as stated.

Acknowledgement. The author wishes to express his gratitude to J. Kincses for his valuable discussions and to $\mathrm{V}$. Totik for encouragement.

\section{References}

[1] J.M. Carnicer and J.M. Peña, On zero-preserving linear transformations, J. Math. Anal. Appl., 266 (2002), 237-258.

[2] H. FAst, Inversion of the Crofton transform for sets in the plane, Real Anal. Exchange, 19 (1993/94), 59-80.

[3] R.J. Gardner, Geometric tomography, Encyclopedia of Math. and its Appl. 58, Cambridge University Press, Cambridge, 2006.

[4] A. Honwitz, Reconstructing a function from its tangent lines, Amer. Math. Monthly, 96 (1989), 807-813. 
[5] R. P. Kanwal, Generalized functions theory and technique, 2nd ed., Birkhäuser, Boston, 1998.

[6] J. Kincses and Á. Kurusa, Can you recognize the shape of a figure from its shadows?, Beitr. Algebra Geom., 36 (1995), 25-34.

[7] J. Kincses, The determination of a convex set from its angle function, Discrete Comput. Geom., 30 (2003), 287-297.

[8] J. Kincses, An example of a stable, even order Quadrangle which is determined by its angle function, Discrete Geometry, in honor of W. Kuperberg's 60th birthday (ed.: A. Bezdek), CRC Press (Marcel Dekker), New York - Basel, 2003, 367-372.

[9] S. G. Krantz and H. R. Parks, The Implicit Function Theorem: History, Theory, and Applications, Modern Birkhäuser Classics, Springer, Basel, 2013.

[10] Á. Kurusa, You can recognize the shape of a figure by its shadows!, Geom. Dedicata, 59 (1996), 103-112; doi: 10.1007/BF00155723.

[11] Á. Kurusa, The shadow picture problem for nonintersecting curves, Geom. Dedicata, 59 (1996), 113-125; doi: 10.1007/BF00181528.

[12] Á. Kurusa, The shadow picture problem for parallel straight lines, J. Geom., 103:3 (2012), 515-518; doi: 10.1007/s00022-012-0137-z.

[13] Á. Kurusa, Visual distinguishability of polygons, Beitr. Algebra Geom., $\quad \mathbf{5 4 : 2}$ (2013), 659-667; doi: 10.1007/s13366-012-0121-7.

[14] Á. Kurusa and T. Ódor, Isoptic characterization of spheres, J. Geom., 106 (2015), 63-73; doi: 10.1007/s00022-014-0232-4.

[15] J. C. C. Nitsche, Isoptic characterization of a circle, (Proof of a conjecture of M.S. Klamkin), Amer. Math. Monthly, 97 (1990), 45-47.

[16] T. J. Richardson, Planar rectifiable curves are determined by their projections, Discrete Comput. Geom., 16 (1996), 21-31.

[17] T. J. Richardson, Total curvature and intersection tomography, Advances Math., 130 (1997), 1-32.

[18] R. Schneider, Functions on a sphere with vanishing integrals over certain subspheres, J. Math. Anal. Appl., 26 (1969), 381-384.

[19] H. Steinhaus, Length, shape, and area, Colloq. Math., 3 (1954), 1-13.

Á. Kurusa, Bolyai Institute, University of Szeged, Aradi vértanúk tere 1, H-6720 Szeged, Hungary; e-mail: kurusa@math.u-szeged.hu 